\title{
Frameworks in Problems of Structural Identification Systems
}

\author{
Nikolay Karabutov \\ Dept. of Problems Control, Moscow technological University (MIREA), Moscow, Russia \\ E-mail: kn22@yandex.ru, nik.karabutov@gmail.com
}

\begin{abstract}
The new approach to structural identification of nonlinear dynamic systems under uncertainty is proposed. It is based on the analysis of virtual frameworks (VF), reflecting a state of a nonlinear part system. Construction VF is based on obtaining special an informational set describing a steady state of a nonlinear dynamic system. Introduction VF demands an estimation of structural identifiability of a system. This concept is as sociated with nonlinearity of system and properties VF. The method of an estimation of structural identifiability is proposed. The appearance of the insignificant virtual frameworks, not satisfying to the condition of structural identifiability, is considered. Algorithms for an estimation of a nonlinearity class on the basis of the analysis of sector sets are proposed. Methods and procedures of the estimation of framework single-valued and multiple-valued nonlinearities are proposed. The method of the structurally-frequency analysis is proposed and applied to validate the obtained solutions. VF is proposed for identification of an order and a spectrum of eigenvalues of a linear dynamic system. The possibility of application VF for the problem solving of identification static systems is shown.
\end{abstract}

Index Terms - Framework, dynamic system, phase portrait, structural identification, nonlinearity, structural identifiability, Lyapunov exponent, structurallyfrequency analysis.

\section{INTRODUCTION. STRUCT URAL APPROACH IN IDENTIFICATION AND CONTROL PROBLEMS}

The structural approach (SA) is widely applied in control theory of nonlinear systems. Explain it with expansion: (i) class of systems which are subject to studying; (ii) design of control algorithms. Processes in such systems have nonlinear character. Special classes of mathematical objects are the basis VF. They allow estimating features and qualitative behavior of nonlinear system. The SA is a dominating direction in control theory after design of methods of the qualitative systems theory. Dominance explains development of the researches related with study of determined chaos, catastrophes, structural dynamics, synchronization [1-4]. Geometrical frameworks are used for the description of processes in such systems. They reflect a change of evolution system in the generalized form. The typical representative of such frameworks is a phase portrait (PP) [3] and its generalization an attractor $[1,4]$. They are most accented reflect features of dynamic systems. Qualitative restructuring PP can be the form of bifurcation, and the steady motion has the form of the attractor [4]. The chaos is a special state of dynamic system. Various mathematical designs (frameworks) are proposed for its description: Smale horseshoe [5], Anosov $y$-system [6] and other representations [2].

Practical worth of the various mathematical designs describing features of nonlinear processes is not always high. Explain it with a lack of experimental information which is the basis for obtaining of these frame works. This is one of the information problems of nonlinear dynamics PP and attractors demand the application of the special means for their construction. Observed variable not always allow obtaining the adequate nonlinear section of a system. Existing difficulties are considered in [7]. They are typical for systems of identification and nonlinear dynamics. Results of work [4] confirm this inference. Authors note that does not exist an optimal method of construction of identification system on experimental data. A successful set of experimental data as it is noted in [4] predetermines efficiency of the identification process mathematical model. Explain it with obtaining of the model describing a system attractor projection. The attractor projection is the basis of the construction an evolution operator.

The chaos has the "destroying" character. But it can play the constructive role in structurization and the organization of the system. So, the chaos [4] can ensure: 1) transition of system to one of the possible attractors; 2) integration of simpler frameworks into complex frameworks; 3) change of regimes in a system. Detection of new properties of the chaos makes activates the further researches of the mechanisms of its appearance. The chaos "breaks" established dynamics of a system. Therefore, Lyapunov exponents (LE) often apply to the analysis of reasons for its cause. $\mathrm{LE}$ is one of the structural performances, allowing tracing a system evolution.

So, geometrical frameworks (GF), though exist complexities, allow solving various problems structurization and estimations of qualitative behavior of a system. They are the basic tool for the analysis of nonlinear dynamics systems. Therefore, we believe that methodological principles of construction GF are identified and areas of their effective application are determined. We understand the structural approach as construction and the analysis of the geometrical frameworks reflecting features of a system. As a rule, GF is mathematical objects which are described by any functions or mappings. General approach 
es to their construction are proposed on the basis of design methods the analysis of stability dynamic systems by means of PP.

Go now to identification systems. Here the concept the structural approach is also widely used. But this concept has meaning unlike nonlinear dynamics. It differs from above stated. Each researcher gives this concept its own interpretation. Methods of structural identification of a various class of systems are considered in [8]. Authors refer to structural identification parametric models of health monitoring and a structural dynamic. The considered algorith ms and procedures are not methods structural identification (structural approach). The term structural dynamics is it is area of research, but it is not structural identification. The same interpretation SA is given in [9].

The problem of identifiability structural autoregressive models with instantaneous effects is considered in [10]. The estimation of Gaussian linear structural models creates problems of system identifiability. Therefore, authors [10] propose to use non-Gaussian models. They will show how to combine non-Gaussian instant model from autoregression models. Authors call such combination of equations structural model of the vector autoregression.

Review [11] contains the analysis of methods identification processes in structural engineering. Various approaches to parametric and nonparametric identification are considered. The interpretation of concept "framework" coincides with a terminology given in two previous paragraphs. The methods of structural identification based on the analysis of a substructure damping are proposed in [12]. Methods of identification parameters nonlinear vibration systems are considered in [13]. The adaptive Kalman filter is a basis of procedures identification. Other approaches to nonlinear identification of structural dynamics are stated in [14]. They are based on an application of traditional identification methods.

So, we see that the problem of structural identification (SI) in most cases is solved on the basis of application the traditional paradig $\mathrm{m}$. It is based on application of the parametric approach and some other procedures (neural network and genetic algorith ms). As a rule, these procedures are variant the parametric approach. The analysis shows that the mathematical frameworks describing a state of nonlinear systems in the form of portraits and mappings, in the theory of structural identification practically were not applied (see below). The analys is of state researches in the field of SI is given in [15]. The main resume which can be made from [15]: direct methods (parametric) are not applicable in SI problems under uncertainty. Therefore, the solution of the SI problem demands design of new approaches. We refer to the m geometrical (structural) methods of synthesis identification systems. The development of such paradigm demands to revise of existing approaches and creation of fundamentally new informational and structural approaches. These approaches should show the framework of a nonlinear dynamic system. This is one of perspective directions of the identification theory development.

Despite the specified difficulties, N. Karabutov [16] has proposed the approach allowing promoting in the problem solution of SI dynamic systems. Following two concepts are the approach basis:

1) introduction of the new variables characterizing framework of the system;

2) construction of virtual frameworks reflecting a state of the nonlinear part system.

Bases of the proposed approach (we will name it $S I$ approach) are stated in [17]. The further develop ment and generalization $S I$-approach on a class of nonlinear static systems is given in $[18,19]$. The analysis and decisionmaking about framework (the mathematical operator describing a system) are performed in special (structural) space. Processes in dynamic systems differ from static systems. Therefore, generalization $S I$-approach on nonlinear dynamic systems is given in [16]. Researches have shown that the concept based on the analysis of virtual frameworks, is efficient for the class of linear dynamic systems (DS).

Next, we state bases $S I$-the approach and we give its generalization and development. We introduce concepts which are necessary for understanding the SI -approach. Methods of auxiliary informational set formation are considered. The informational set is basis of the problem solution creation of virtual frameworks $S_{e y}$.

Introduction $S_{e y}$ demanded design of methods estimation of a structural identifiability nonlinear DS. Structural identifiability is based on the parametric identifiability and specifies demands to the form $S_{e y}$. Criteria for decision making about a class of the functions describing a nonlinear part of the dynamic system are presented. Algorithms of SI nonlinear dynamic system are considered. We show how the $S I$-approach can be applied to the estimation of the framework linear DS. The final part of the work contains the application $S I$-approach to static systems. We do not use the term "the structural approach" as it does not coincide with the proposed methodology.

\section{PRoblem St ATEMENT OF StRUCTURAL IDENTIFICATION NONLINEAR SYSTEMS}

Consider dynamic system

$$
\begin{aligned}
& \dot{X}=F(X, U, A, t), \\
& Y=F_{Y}(X, U, A, t),
\end{aligned}
$$

where $U \in \Omega_{U} \subset \mathrm{U} \subseteq R^{m}, Y \in \mathrm{Y} \subset R^{n}$ are measured input and output, $X \in \mathrm{X} \subseteq R^{q}$ is state vector, $\mathrm{Y} \subseteq \mathrm{X} \subseteq R^{n}, F: R^{q} \times R^{m} \times J \rightarrow R^{q}$ is smooth continuously differentiable on $X$ and $A$ a vector function, $A \in R^{q \times q}$ the matrix of parameters, $t \in J \subset R$, $F_{Y}: R^{q} \times R^{m} \times J \rightarrow R^{n}$ is a function specifying a mode of formation the systemoutput.

Set of the measured data

$$
\mathrm{I}_{o}=\mathrm{I}(U, Y)=\left\{\tilde{U} \in R^{m}, \tilde{Y} \in R^{n}, t \in J\right\}
$$


observed on a time gap $J$.

Problem: make the decision about a structure operator $F$ in (1) on the basis of the analysis and processing of the set $\mathrm{I}_{o}$.

\section{STRUCT URAL METHODS IN IDENT IFICATION SYSTEMS OF NONLINEAR DYNAMIC SYSTEMS}

\section{A. Approach to Identification of Class Nonlinearities}

State the approach to structural identification for the system(1) with the selected linear part

$$
\begin{aligned}
& \dot{X}=A X+\varphi(y) I+B u, \\
& y=C^{T} X,
\end{aligned}
$$

where $u \in R, y \in R$ are input and output; $A \in R^{q \times q}$, $B \in R^{q}, I \in R^{q} \quad C \in R^{q}$ are matrixes; $\varphi(y)$ a some scalar nonlinear function. $A$ is Hurwitz matrix.

Different assumptions can become about function structure $\chi=\varphi(y)$. They are determined by a level of the a priori information. The methods based on procedures of linearization [20], are applied at a priori determinacy. The following assumption [21] about $\varphi(y)$ makes at research of the absolute stability of nonlinear systems

$$
\chi \in F_{\varphi}=\left\{\varphi(\xi) \xi \geq \xi^{2}, \xi \neq 0, \varphi(0)=0\right\}
$$

where $\xi \in R$ is input nonlinear element. We assume that $\xi$ is the linear combination of state variables.

The sector condition is often used for an approximation $\chi$. The sector is defined as the area restricted to two straight lines between which nonlinear function lies

$$
\begin{gathered}
\chi \in F_{\varphi}=\left\{\gamma_{1} \xi^{2} \leq \varphi(\xi) \xi \leq \gamma_{2} \xi^{2}, \xi \neq 0\right. \\
\left.\varphi(0)=0, \gamma_{1} \geq 0, \gamma_{2}<\infty\right\}
\end{gathered}
$$

Static nonlinearities are often applied in control systems. Further we suppose that the model described by a static (algebraic) equation, is applicable for the estimation of the function $\varphi(\cdot)$. Generate corresponding subset of the measured data or their transformed analogue that such model to identify.

The set $I_{o}$ for system (3) has the form

$$
\mathrm{I}_{o}=\left\{u(t), y(t), t \in J=\left[t_{0}, t_{k}\right]\right\}
$$

Problem: estimate a class of nonlinear functions $\varphi(y)$ in (3) and characteristics a matrix $A$ on the basis of the data processing (6).

Apply the informational synthesis $[17,18]$ to the problem solution. It realizes following steps.
1. Obtaining of a set $\mathrm{I}_{N, g}$ containing the data about the nonlinear part of the system(3).

2. Construction of virtual frameworks reflecting nonlinear properties of the system(3).

3. The design of algorithm decision making about the class nonlinearity $F_{\varphi}$.

4. The design of procedure for nonlinearity identification in the class $F_{\varphi}$.

5. The design of a method of decision-making about the nonlinearity form in (3).

6. An estimation of a dimension and a spectrum of eigenvalues the matrix $A$.

These steps are based on construction and the analysis of virtual structures. Form and properties VS depend on the system(3).

\section{B. Formation of Set $\mathrm{I}_{N, g}$}

The method of construction set $\mathrm{I}_{N, g}$ is based on results of the work [16]. Apply differentiation operation to $y(t)$ and designate the obtained variable as $x_{1}$. The introduction $x_{1}$ gives the expansion of the informational set $\mathrm{I}_{o}$ : $\mathrm{I}_{\text {ent }}=\left\{\mathrm{I}_{o}, x_{1}\right\}$.

Remark 1. If variables $u, y$ are measured with an error, then apply procedure of filtering or smoothing.

Consider the first step of the informational synthesis. Generate a subset $\mathrm{I}_{g} \subset \mathrm{I}_{\text {ent }}$ corresponding to the particular solution of the system (3) (to the steady state). We form set $\mathrm{I}_{g}$, excepting the data $\mathrm{I}_{t r}$. $\mathrm{I}_{t r}$ contains the information about the transient process in the system. Then $\mathrm{I}_{g}=\mathrm{I}_{e n t} \backslash \mathrm{I}_{t r}$. Apply mathematical model

$$
\hat{x}_{1}^{l}(t)=H^{T}\left[\begin{array}{lll}
1 & u(t) & y(t)
\end{array}\right]^{T}
$$

to a selection of a linear component in $x_{1}$. The variable $x_{1}$ is defined on the interval $J_{g}=J \backslash J_{t r}$. Here $H \in R^{3}$ is the vector of parameters.

Determine the vector $H$ as

$$
\left.\min _{H} Q(e)\right|_{e=\hat{x}_{1}^{l}-x_{1}} \rightarrow H_{o p t} .
$$

where $Q(e)=0.5 e^{2}$.

Find the prediction for the variable $x_{1}$ on the basis of the model (7) $\forall t \in \mathrm{I}_{g}$ and form an error $e(t)=\hat{x}_{1}^{l}(t)-x_{1}(t)$. $e(t)$ depends on the nonlinearity $\varphi(y)$ in the system (3). Then we obtain set $\mathrm{I}_{N, g}=\left\{y(t), e(t) t \in J_{g}\right\}$ which we use at the second stage of the informational synthesis. Apply the designation $y(t)$, supposing that $y(t) \in \mathrm{I}_{N, g}$. 
Remark 2. The choice of structure the model (7) is one of the stages of the structural identification. Results of modeling show that the model (7) is applicable in identification systems of objects with static nonlinearities. The problem of the choice the structure model (7) demands the further research for other classes of nonlinearities. Next, we set an example of the problem solution for one class of systems.

The further problem solution of SI is based on the analysis of frameworks $S_{e y}, S_{e k}$ reflecting the state of the nonlinear part of the system(3).

\section{Frameworks $S_{e y}, S_{e k}$}

Virtual frameworks have proposed in [17] for the analysis and synthes is of identification systems. Development and generalization VS on a class of static systems is given in [22]. We describe the approach to construction VS for dynamic systems.

Let $S$ is a phase portrait of the system(3) described by a function $x_{1}=f_{S}(y)$. We will study the phase portrait of the system (3) in space $\mathscr{P}_{y e}=(y, e)$. Name $\mathscr{P}_{y e}$ as the structural space. The framework $S_{e y}$ described by function $\Gamma_{e y}:\{y\} \rightarrow\{e\} \forall t \in J_{g}$, is the phase portrait of the nonlinear part of the system (3). $S_{e y}$ can be closed. It is a characteristic property $S_{e k}$ of dynamic systems. We will apply to decision-making also $S_{e k}$-framework which is described by the function $\Gamma_{e k}:\left\{k_{s}(t)\right\} \rightarrow\{e(t)\}$, where $k_{s}(t) \in R$ is the factor of structural properties [17, 22]

$$
k_{s}(t)=\frac{e(t)}{y(t)}
$$

Example 1. Consider the system (3) second order with

$$
\begin{aligned}
& \varphi(y)=\left\{\begin{array}{r}
2.5, \text { if }((y-d)>\bar{v}) \&\left(y^{\prime}>0\right), \\
\alpha(y-d), \text { if }((y-d) \leq \bar{v}) \&\left(y^{\prime}>0\right), \\
1.5, \text { if }((y-d) \leq \underline{v}) \&\left(y^{\prime}>0\right), \\
2.5, \text { if }(y>\bar{v}) \&\left(y^{\prime} \leq 0\right), \\
\alpha y, \text { if }(y<\bar{v}) \&\left(y^{\prime} \leq 0\right), \\
1.5 y, \text { if }(y<\underline{v}) \&\left(y^{\prime} \leq 0\right),
\end{array}\right. \\
& A=\left[\begin{array}{cc}
0 & 1 \\
-3 & -4
\end{array}\right], y(0)=x(0)=3.5, y^{\prime}(0)=x^{\prime}(0)=2 \\
& u(t)=5-5 \sin (0.1 \pi t), y=x,
\end{aligned}
$$

where $d=1, \bar{v}=2.5, \underline{v}=1.5, \alpha=1$.

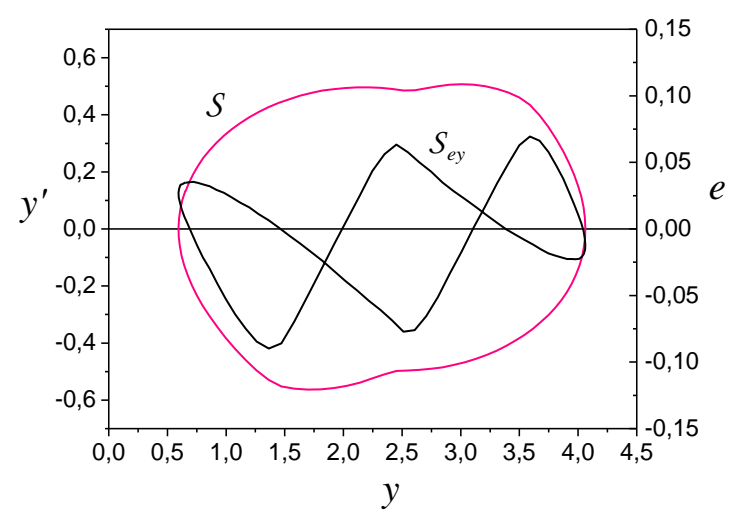

Fig.1. Frameworks of the second order system with the function $\varphi(y)(8)$.

Show in Fig. 1 the phase portrait $S$ and the framework $S_{e y}$ describing the steady state of the system. We see (Fig. 1) that the framework $S$ is virtually not informative. The framework $S_{e y}$ confirms the nonlinearity of the system. $S_{e y}$ is obtained on the basis of the model (7) with $H=\left[\begin{array}{lll}0.22 & 0.23-0.59\end{array}\right]^{T}$.

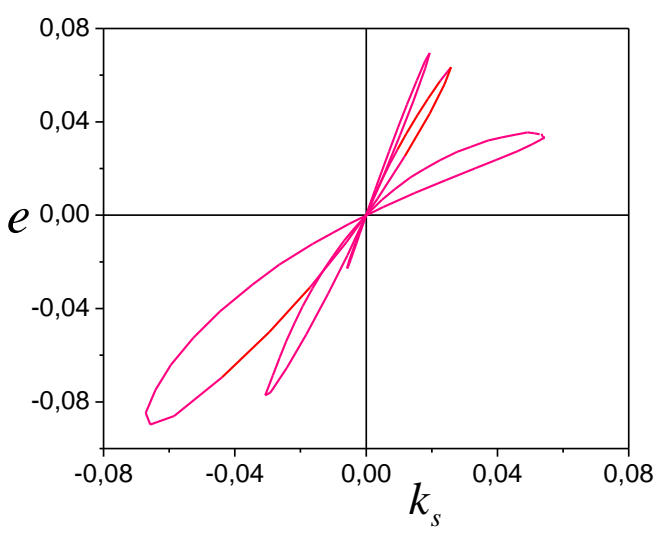

Fig.2. Framework $S_{e k}$.

Show framework $S_{e k}$ in Fig. 2.

We see that the form and properties $S_{e y}$ influence on the identifiability of nonlinear dynamic systems. Therefore, we will consider properties of the set $\mathrm{I}_{N, g}$ on which the framework $S_{e y}$ is specified.

\section{About Properties $\mathrm{I}_{N, g}$. Structural Identifiability of System (3)}

Consider the properties of set $\mathrm{I}_{N, g}$ ensuring the problem solution of the structural identification. At first, the set $\mathrm{I}_{o}$ should ensure the solution of parametric identification problem the model (7). This means that the input $u(t)$ should be non-degenerate and constantly excited on an interval $J$. Secondly, the input $u(t)$ should ensure informative framework $S_{e y}\left(\mathrm{I}_{N, g}\right)$ (or $S_{e k}$ ), securing deci 
sion-making about nonlinear properties of the system (3). Name an input representative if it allows on the basis of the analysis $S_{e y}$ make the decision about properties of the system.

Let the framework $S_{e y}$ is closed. This to mean that the area $S_{e y}$ is not null. Designate altitude $S_{e y}$ as $h\left(S_{e y}\right)$. $h\left(S_{e y}\right)$ is the distance between two points of the opposite sides framework $S_{e y}$.

Statement 1 [16]. Let: 1) the linear part of system (3) is stable, and nonlinearity satisfies to the condition $(5) ; 2$ ) the input $u(t)$ is restricted, piecewise continuous and constantly excited; 3) exists such $\delta_{S}>0$ that $h\left(S_{e y}\right) \geq \delta_{S}$. Then the framework $S_{e y}$ is identified on the set $\mathrm{I}_{N, g}$.

Further, we suppose that $S_{e y}$ has the specified property.

We will name the framework $S_{e y}$ having the specified properties $h$-identified. Further, we suppose that $S_{e y}$ is $h$-identified.

Consider features of concept $h$-identifiability.

1. $h$-identifiability is a concept not parametric, but structural identification.

2. The demand of the parametric identifiability is the basis $h$-identifiability.

3. $h$-identifiability makes more rigid demands to the systeminput.

Feature 3 means that "the bad" input can satisfy condition of the constancy excitation. Such input can give socalled "insignificant" $S_{e y}$-structure ( $\mathcal{N} S_{e y}$-framework). But the $\mathcal{N} S_{e y}$-structure can be $h$-identified. Property of insignificance under uncertainty gives identification of nonlinearity, atypical for an examined system.

Consider existence conditions of $\mathcal{N} S_{e y}$-structures. Consider a class of nonlinear functions to which homotopy operation is applicable. The homotopy [23] is the operation of obtaining of one part of a geometrical figure from another part on the basis of its rotation and extension about a certain point on a plane $(y, e)$.

Consider the framework $S_{e y}$. Let $S_{e y}=F_{S_{e y}}^{l} \cup \mathcal{F}_{S_{e y}}^{r}$, where $F_{S_{e y}}^{l}, F_{S_{e y}}^{r}$ are left and right fragments $S_{e y}$. Determine for $F_{S_{e y}}^{l}, F_{S_{e y}}^{r}$ secants

$$
\gamma_{S}^{l}=a^{l} y, \gamma_{S}^{r}=a^{r} y
$$

where $a^{l}, a^{r}$ are the numbers computed by means of the least-squares method (LSM).

Theorem 1 [7]. Let: 1) the framework $S_{e y}$ is $h$ - identified; 2) the framework $S_{e y}$ has the form $S_{e y}=F_{S_{e y}}^{l} \cup F_{S_{e y}}^{r}$, where $F_{S_{e y}}^{l}, F_{S_{e y}}^{r}$ are left and right fragments $S_{e y}$; 3) secants for $F_{S_{e y}}^{l}, F_{S_{e y}}^{r}$ have the form (9). Then $S_{e y}$ is $\mathcal{N} S_{e y}$-structure, if

$$
\left|a^{l}-a^{r}\right|>\delta_{h}
$$

where $\delta_{h}>0$ is some specified number.

Remark 3. The theorem 1 can be proved on the basis of the homotopy of sets [24]. Estimate proximity of sets $F_{S_{e y}}^{l}, F_{S_{e y}}^{r}$ in this case. The approach based on secant method is simpler in implementation.

Remark 4. $\mathcal{N S}_{e y}$-structures are characteristic for systems with multiple-valued nonlinearities. They are the result of the inadequate application of input actions.

Definition 1. If the framework $S_{e y}$ is $h$-identified and the condition $\left|a^{r}-a^{r}\right| \leq \delta_{h}$ is satisfied, then $S_{e y}$ is structurally identified or $h_{\delta_{h}}$-identified.

Let the framework $S$ has $m$ features. We will understand features of a function $f$ as continuum loss on some interval $\mathrm{I}_{y}^{j}$, flex points of function or extremes. These features are an indication of nonlinearity $f$.

Apply the model (7) and construct the framework $S_{e y}$ in the space $\mathscr{P}_{y e}$.

Definition 2. Model (7) is $S M$-identifying if the framework $S_{e y}$ is $h_{\delta_{h}}$-identified.

Theorem 2. Let: (i) the input $u(t)$ is constantly excited and ensures $h_{\delta_{h}}$-identifiability of the system (3); (ii) the phase portrait $S$ of the system (3) contains $m$ features; (iii) the $S_{e y}$-structure is $h_{\delta_{h}}$-identified and contains the fragments corresponding to features of the phase portrait $S$. Then model (7) is $S M$-identifying.

Proof. The phase portrait $S$ is $h_{\delta_{h}}$-identifying. Model (7) is linear. Input $u(t)$ is constantly excited and ensures $h_{\delta_{h}}$-identifiability of the system(3). If the model (7) adequately describes the change of the linear part of the system (3) the variable $e$ reflects features $S$. Adequacy of the model is determined by the specified structure of the equation (7) on set $\mathrm{I}_{o}\left(t \in \bar{J}_{g}\right)$, where $\bar{J}_{g}$ is definition domain $S$. Since the $S_{e y}$-structure is $h_{\delta_{h}}$-identified, and the equation (7) gives a mode of its obtaining the model is adequate and, hence, $S M$-identifying.

The theorem 2 shows that if model (7) not SM identifying it is necessary to change structure of the model (7) or an informational set for its construction. 
Consider one more approach to the estimation $h_{\delta_{h}}$ identifiability of the system(3).

Problem: design on the basis of the processing $\mathrm{I}_{N, g}$ the integral indicator allowing makes decision about the insignificance $S$ framework.

Approaches based on a principle of a covering are proposed $^{1}$ for an estimation of dimension (capacity) of an attractor in dynamic systems and fractals. The simplified inde $x$ is a topological dimension. It estimates geometry of a framework and it does not always reflect its interior features. Attractors and fractals often are inhomogeneous. Inhomogeneity reflects nonuniformity of distribution points on the framework (fractal). Estimations of the frameworks inhomogeneity obtain by means of parameters reflecting properties of the system. The inhomogeneity reason is different probabilities of filling geometrically equal elements of the fractal. Inhomogeneity generally is mis match between probabilities of fractal filling the specified objects and geometrical sizes of corresponding are$\mathrm{as}^{2}$. Such inhomogeneous fractal objects name mult ifractals. $S_{e y}$-structures of dynamic systems with multiplevalued nonlinearities are an example of inhomogeneous frameworks.

Various indexes of a covering (correlation dimension, the informational dimension, and etc.) are approached and time-consuming. They do not always give an estimation of a geometrical difference of framework frag ments. Therefore, we introduce the integral performance of the $S$-framework. It is the distribution function of the variable $e$. Such approach eliminates various a priori assumptions concerning the framework covering local objects. The proposed approach is based on a method of the structurally-frequency analysis which is considered in section 3.F.

So, consider the system (3). Obtain for it the framework $S_{e y}$. Fulfil the fragmentation $S_{e y}$. Fragmentation conditions depend on the form $S_{e y}$. We have $S_{e y}=F_{S_{e y}}^{l} \cup F_{S_{e y}}^{r}$, where $F_{S_{e y}}^{l}, F_{S_{e y}}^{r}$ are left and right parts of the framework $S_{e y}$. Functions describe fragments $F_{S_{e y}}^{l}, F_{S_{e y}}^{r}$, where $\left\{e^{l}\right\} \subseteq\{e\},\left\{e^{r}\right\} \subseteq\{e\}$. Construct for $F_{S_{e y}}^{l}, F_{S_{e y}}^{r}$ frequency distribution functions (histogram) $\mathcal{H}^{l}, \mathcal{H}^{r}$. Obtain on the basis $\mathcal{H}^{l}, \mathcal{H}^{r}$ distribution integral functions $I \mathcal{H}^{l}, I \mathcal{H}^{r}$. Let $\mathrm{I}_{\mathcal{H}}=\{i \Delta e, i=\overline{1, k}\}$ is a definition range of functions $\mathcal{H}^{l}, \mathcal{H}^{r}$. Describe range of values of functions $I \mathcal{H}^{l}, I \mathcal{H}^{r}$ by vectors

$$
L\left(I \mathcal{H}^{l}\right)=\left[I \mathcal{H}_{1}^{l}, I \mathcal{H}_{2}^{l}, \ldots, I \mathcal{H}_{k}^{l}\right]^{T}
$$

\footnotetext{
${ }^{1}$ J. Feder, Fractals. New York, Plenum Press, 1988.

2 S.V. Bozhokin, D.A. Parshin, Fractals and Multifractals; Scientific Publishing Centre I"Regular and Chaotic Dynamicsl": Moscow-Izhevsk, 2001 .
}

$$
R\left(I \mathcal{H}^{r}\right)=\left[I \mathcal{H}_{1}^{r}, I \mathcal{H}_{2}^{r}, \ldots, I \mathcal{H}_{k}^{r}\right]^{T}
$$

Here $k$ is the number of the pockets specified on $\mathrm{I}_{\mathcal{H}}$, $\Delta e$ is a magnitude of the pocket.

Apply the model

$$
\hat{R}=a_{H} L\left(I \mathcal{H}^{l}\right)
$$

and determine the parameter $a_{H}$ by means of LSM.

The model is adequate, if parameter $a_{H} \in O(1)$, where $O(1)$ is a neighbourhood 1 . If the condition $a_{H} \in O(1)$ is true, the system (3) is $h_{\delta_{h}}$-identified and $S_{e y} \neq \mathcal{N S} S_{e y}$. Otherwise, the framework $S_{e y}$ is insignificant.

So, statement is true.

Statement 2. Let: 1) the framework $S_{e y}$ is obtained for system (3); 2) the framework $S_{e y}$ has the form $S_{e y}=F_{S_{e v}}^{l} \cup F_{S_{e v}}^{r}$, where $F_{S_{e v}}^{l}, F_{S_{e v}}^{r}$ are fragments framework defined on set $\{y(t)\} ; 3$ ) frequency distribution functions $\mathcal{H}^{l}, \mathcal{H}^{r}$ and cumulative frequency functions $I \mathcal{H}^{l}, I \mathcal{H}^{r}$ are obtained for $\left.F_{S_{e y}}^{l}, F_{S_{e y}}^{r} ; 4\right)$ the function $R\left(I \mathcal{H}^{r}\right)=a_{H} L\left(I \mathcal{H}^{l}\right)$ is known. Then the system (3) is $h_{\delta_{h}}$-identified, if $a_{H} \in O(1)$.

Introduce $D_{h}$-dimension of the system (3) by analogy to fractals.

Definition 3. The system(3) has dimension $D_{h}=a_{H}$ if its is $h_{\delta_{h}}$-identified.

Definition 3 shows that dimension of a structurally identified system is approximate to 1 .

Example 2. Consider an example 1 of section 3. C. Let $u(t)=3-2 \sin (0.1 \pi t)$. Obtain structures $S, S_{e y}$ and show them in Fig. 3.

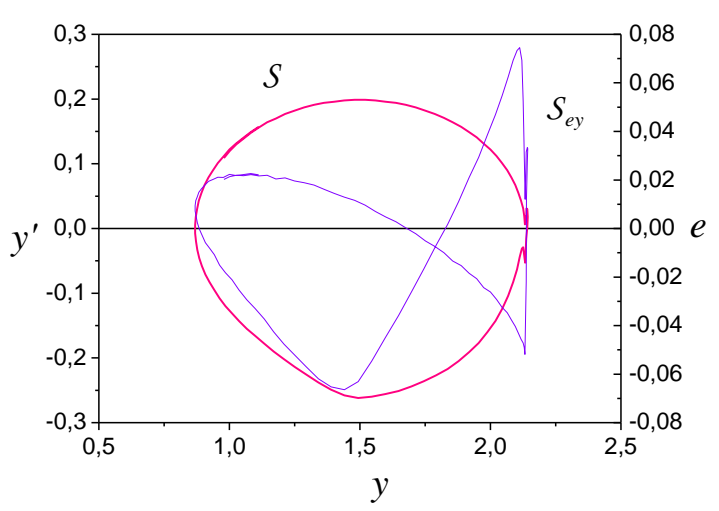

Fig.3. Structurally non-identifiable system of the second order with nonlinearity (8) 
The Fig. 3 shows that frameworks $S$ and $S_{e y}$ are asymmetrical concerning the straight line $y=1.5$. Frameworks $S, S_{e y}$ are $h$-identified, but they are not $h_{\delta_{h}}$-identified. Asymmetry shows the difference between the left and right parts of frameworks. So, the system is structurally not identifiable. $S_{e y}$ is the example $\mathcal{N} S_{e y}$ structure. Explain such behavior of the system an inadequate choice of the input $u(t)$.

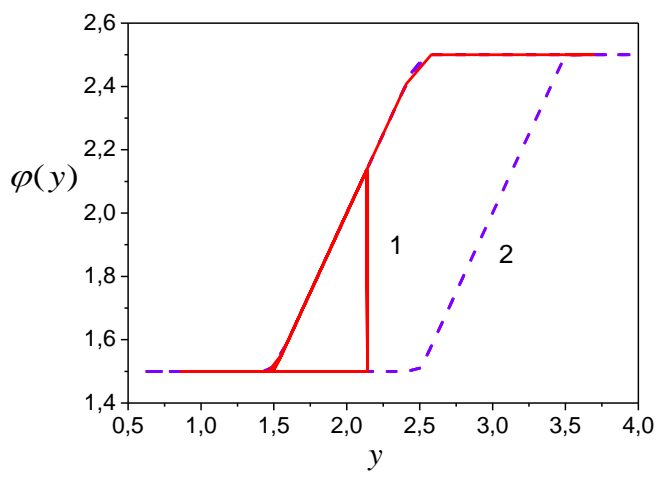

Fig.4. Defectiveness $\varphi(y)$.

Divide the framework $S_{e y}$ the straight line $y=1.5$ into two fragments $F_{S_{e y}}^{l}, F_{S_{e y}}^{r}$. Secants (9) are determined for frag ments $F_{S_{e y}}^{l}, F_{S_{e y}}^{r}$ with $a^{l}=-0.038, a^{r}=0,041$. Let $\delta_{h}=0.02$. Apply the theorem 1 and obtain that $S_{e y}$ is $\mathcal{N S} S_{e y}$-structure. Confirmation of defectiveness the function $\varphi(y)$ gives Fig. 4. Curves 1 and 2 in Fig. 4 are "a defective" and the reference hysteresis $\varphi(y)$.

Let $u(t)=5-5 \sin (0.1 \pi t)$. Obtain frameworks $S_{e y}, S$ and show them in Fig. 1 . We see that the system is $h_{\delta_{h}}$ identified. We have applied model (7) with the vector $H=[0.220 .23-0.59]^{T}$ to obtaining the $S_{e y}$-structure. So, $S_{e y} \neq \mathcal{N} S_{e y}$. This inference confirms that the model is $S M$-identifying.

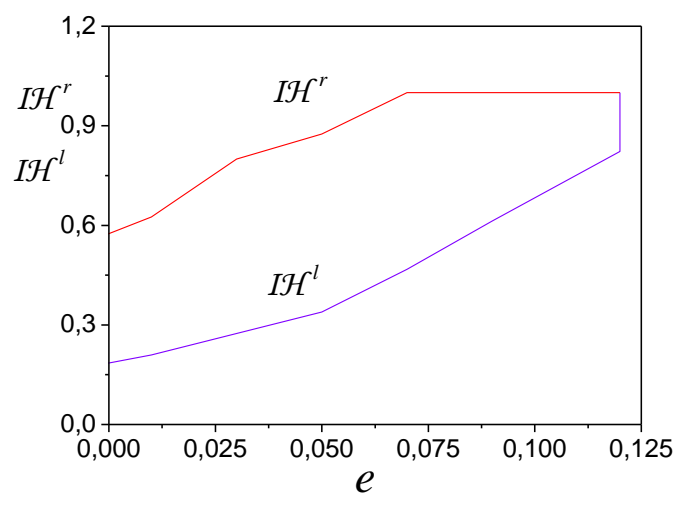

(a) cumulative frequency functions $I \mathcal{H}^{l}, I \mathcal{H}^{\prime}$

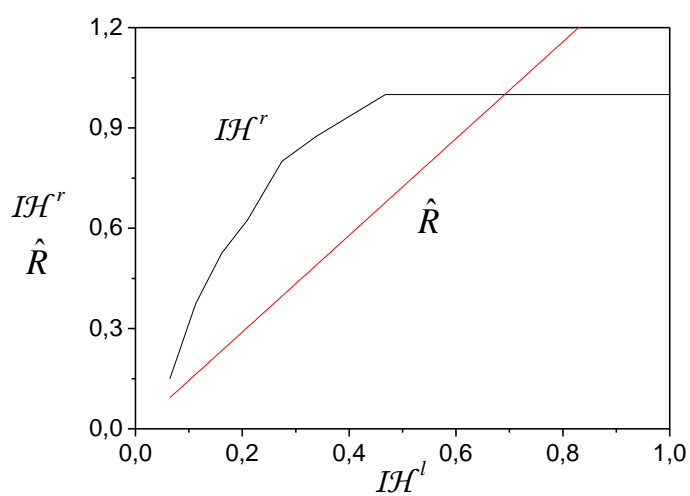

(b) the estimation of structural identifiability of the system (3)

Fig.5. The estimation of the system structural identifiability of by means of the structurally-frequency analysis.

Fulfil check of results by means of the structurallyfrequency analysis. Obtain for $F_{S_{e v}}^{l}, F_{S_{e y}}^{r}$ function $I \mathcal{H}^{l}, I \mathcal{H}^{r}$ (Fig. 5).

The factor $a_{H}$ of the model $\hat{R}=a_{H} L\left(I \mathcal{H}^{l}\right)$ (Fig. 5(b)) is equal 1.447. The coefficient of determination is equal 0.9. This inference confirms Fig. 5 (a). We see the difference between $I \mathcal{H}^{l}, I \mathcal{H}^{r}$. Hence, the structurally-frequency analysis confirms that $S_{e y}=\mathcal{N} S_{e y}$.

Go now to an estimation of a class of nonlinearity $\varphi(y)$. It is one of the main problems of the structural identification.

\section{E. Estimation of Nonlinearity Class}

Consider classes of single-valued $F_{o v}$ and multiplevalued $F_{m v}$ nonlinearities. These classes contain many nonlinear functions. We will describe the approach to identification of the nonlinearity class $[15,16]$ based on the analysis of sector sets.

Fulfil the fragmentation of the framework $S_{e y}$, using a subset $\mathrm{I}_{\varphi} \subseteq \mathrm{I}_{N, g}$. $\mathrm{I}_{\varphi}$ reflects the change of the function $\chi=\varphi(y)$ in the structural space $\mathscr{P}_{y e}$. The problem of obtaining of a subset $\mathrm{I}_{\varphi}$ is non-trivial and depends on the a priori information. Consider the case of absence a priori information. The problem is reduced to the selection of the change interval the variable $y \in \mathrm{I}_{\varphi}$ on which we see features of function $\chi$. Select the subset $\mathrm{I}_{\varphi}$ on the basis of the analysis of change the framework $S_{e y}$. The fragment $F R_{\varphi} \subset S_{e y}$ corresponds $\mathrm{I}_{\varphi}$. Use fragments $F R_{\varphi} \subset S_{e y}$ in which features of the change $S_{e y}$ are reflected.

Select on the basis of the analysis $F R_{\varphi} \subset S_{e y}$ set of candidates as $\mathrm{I}_{y}^{j} \subset \mathrm{I}_{\varphi}(j=\overline{1, s}) . \mathrm{I}_{y}^{j}$ can contain not associated with features of the nonlinear function of subsets. Name the set $\mathrm{I}_{y}^{j}$ informative [18] if it allows detecting these features. 
Statement 3. Let functions

$$
\left(\mu_{e}^{j}(t)=\frac{d e(t)}{d y}\right) \vee\left(\mu_{k_{s}}^{j}(t)=\frac{d k_{s}(t)}{d y}\right) \forall j=\overline{1, s}
$$

on some interval $\mathrm{I}_{y}^{j}$ are not continuous or in their behavior there are features. Then the interval $\mathrm{I}_{y}^{j}$ is the informative.

Such approach is effective under a priori uncertainty. The specified property of self-descriptiveness depends on $h$-identifiability of the framework $S_{e y}$. Except not informative subsets $I_{y}^{j}$ on the basis of the analysis of changing the indexes $\mu_{e}^{j}, \mu_{k_{s}}^{j}$. Include on remained $\mathrm{I}_{y}^{j}$ in composition $\mathrm{I}_{\varphi}$

$$
\mathrm{I}_{\varphi}=\bigcup_{i}^{v} \mathrm{I}_{\varphi}^{i}\left(\mathrm{I}_{y}^{i}\right), v \leq s .
$$

So, the informational set $\mathrm{I}_{\varphi} \subseteq \mathrm{I}_{N, g}$ is obtained. Go to the estimation of nonlinearity class on the basis of the analysis $\mathrm{I}_{\varphi}$.

Consider the fragment $F R_{\varphi}^{i} \subset S_{e y}$ defined on $\mathrm{I}_{\varphi}^{i}$ for $\exists i \geq 1$. Construct for $F R_{\varphi}^{i}$ a sector set [15]. Apply the least-squares method and determine for $\mathcal{F R}_{\varphi}^{i}$ on $\mathrm{I}_{\varphi}^{i}$ the secant

$$
\bar{\gamma}_{i}=\bar{\gamma}(y(t))=a_{i} y(t)+b_{i},
$$

Determine a mean value $\bar{y}_{i}$ for $y(t)$ on $\mathrm{I}_{y}^{i} \subset \mathrm{I}_{\varphi}$. Let $\bar{y}_{i}$ is a centre $F R_{\varphi}^{i}$ on $\mathrm{I}_{y}^{i} \subset \mathrm{I}_{\varphi}$. Draw a perpendicular from the point $\bar{y}_{i}$ before intersection with $\bar{\gamma}_{i}$ on the plane $(y, e)$. Set the magnitude $c_{i}>0$ and in the point $\alpha=\left(\bar{y}_{i}, e\left(\bar{y}_{i}\right)\right)$ construct straight lines

$$
\bar{\gamma}_{i,-}=a_{i,-} y(t)+b_{i}, \bar{\gamma}_{i,+}=a_{i,+} y(t)+b_{i},
$$

where $a_{i,+(-)}=a_{i} \pm c_{i}$. Let $\operatorname{Sec}_{a_{i}}\left(F R_{\varphi}^{i}\right)=\left(\bar{\gamma}_{i,-}, \bar{\gamma}_{i,+}\right)$ is the sectorset for $F R_{\varphi}^{i}$ and

$$
\operatorname{Sec}\left(F R_{\varphi}^{i}\right)=\operatorname{Sec}_{\alpha, l}\left(F R_{\varphi}^{i}\right) \cup \operatorname{Sec}_{\alpha, r}\left(F R_{\varphi}^{i}\right),
$$

where $\operatorname{Sec}_{\alpha, l}\left(F R_{\varphi}^{i}\right), \operatorname{Sec}_{\alpha, r}\left(F R_{\varphi}^{i}\right)$ are subsets $\operatorname{Sec}\left(F R_{\varphi}^{i}\right)$ located more to the left and more to the right of the point $\alpha$.

Construct secants

$$
\bar{\gamma}_{i, l}=a_{i, l} y(t)+b_{i, l}, \quad \bar{\gamma}_{i, r}=a_{i, r} y(t)+b_{i, r}
$$

for each of parts $F R_{\varphi, l(r)}^{i}$ of the frag ment $F R_{\varphi}^{i}$, belonging $\operatorname{Sec}_{\alpha, i}\left(F R_{\varphi}^{i}\right)$ and $\operatorname{Sec}_{\alpha, r}\left(F R_{\varphi}^{i}\right)$. Apply modification of the statement from [15].

Let exists such $\delta_{i}>0$ that

$$
\left|a_{i, l}-a_{i}\right| \leq \delta_{i}, \quad\left|a_{i, r}-a_{i}\right| \leq \delta_{i} .
$$

Theorem 3 [15]. Let for system (3) in space $\mathscr{P}_{y e}=(y, e):$ (i) frame works $\mathcal{F R}_{\varphi, r}^{i}, \mathcal{F R}_{\varphi, l}^{i}$ described by mappings $\Gamma_{e y, l(r)}:\{y\}_{i, l(r)} \rightarrow\{e\}_{i, l(r)}$, and secants (11) corresponding to it are obtained; (ii) the frag ment $F R_{\varphi}^{i}$ has the secant (12), where $\{y\}_{i, l(r)} \subset \mathrm{I}_{\varphi}^{i},\{e\}_{i, l(r)} \subset \mathrm{I}_{\varphi}^{i}$. Then: 1) function $\varphi(y) \in \mathcal{F}_{o v}$ if it is fulfilled (13); 2) function $\varphi(y) \in F_{n v}$ if (13) it is not fulfilled.

The theorem 3 shows if conditions (13) are satisfied, then the condition of Holder-Lipchitz is fair for $\varphi(y)$ and homotopy operation is applicable to sectors $\operatorname{Sec}_{\alpha, l}\left(F R_{\varphi}^{i}\right), \operatorname{Sec}_{\alpha, r}\left(F R_{\varphi}^{i}\right)$.

Remark 5. Frameworks $S_{e y}$ and $S_{e k}$ are applicable for estimation of nonlinearity parameters.

\section{F. Estimation of Nonlinearity Structure}

The problem of structural identification of nonlinear systems under uncertainty is complicated. The general approach is not developed for its solution. Each class of nonlinearities has the features. They are reflected in the behavior of trajectories of a system. Detection of these features under uncertainty gives the analysis $S_{e y}$ or $S_{e k}$. We will describe estimation procedures of the structure $\varphi(y)$, despite the specified difficulties. They are based on a phenomenological analysis $S_{e y}$. The nonlinearity class influences of identification procedure the function $\varphi(y)$. Identification is performed in the structural space $\mathbb{P}_{y e}=(y, e)$. This feature influences in design of estimation structure method. Further we give the development of results obtained in [16].

Class $F_{o v}$

We introduce a series of concepts [16]. Further, we propose a criterion of decision making. Then we synthesize the structure $\varphi(y)$ satisfying to this criterion. The solution is based on the application of the theorem 3.7 [24].

Let $\varphi(y) \in F_{o v}$. Construct for $i-$ th fragment $F_{\varphi, k}^{i} \subset S_{e k}$ the secant described by the polynomial

$$
\bar{\gamma}_{k, p}^{i}\left(e, k_{s}\right)=\bar{\gamma}_{k, p}^{i}=a_{0, p}^{i}+\sum_{j=1}^{p_{i}} a_{1, j, p}^{i} k_{s}^{j}
$$

where $k_{s}(t)=e(t) / y(t)$. 
Determine the order of the polynomial (14) of the condition

$$
\max _{j} r_{e \bar{\gamma}_{k, p}^{i}}^{2}(j) \rightarrow p_{i}^{*}
$$

where $r_{e \bar{\gamma}_{k, p}^{i}}^{2}$ is a coefficient of determination between $e$ and $\bar{\gamma}_{k, p}^{i}$.

Consider a class of elementary functions

$$
F_{P_{f}}=\left\{P_{f_{i}} \in F_{P_{f}}, i=\overline{1, \# F_{P_{f}}}\right\} .
$$

Construct on the basis $P_{f_{i}} \in F_{P_{f}}$ the secant for the fragment $F R_{\varphi, k}^{i} \subset S_{e k}$

$$
\bar{\gamma}_{k, P_{f_{n}}}^{i}\left(e, k_{s, i}\right)=\bar{\gamma}_{k, P_{f_{n}}}^{i}(t)=a_{k, P_{f_{n}}}^{i} k_{s, n}\left(t, P_{f_{n}}\right),
$$

$n=\overline{1, m_{T_{P_{f}}}}$, and compute the coefficient of determination $r_{e k, i, P_{f_{n}}}^{2}$, where $k_{s, i}\left(t, P_{f i}\right)=e(t) / P_{f_{i}}(t), m_{T_{P_{f}}}=\# F_{P_{f}}$ is a cardinal number of the class $F_{P_{f}}$.

Let

$$
\delta_{*}^{2}=r_{e k, i, P_{f_{n}}}^{2}\left(p_{i}^{*}\right)
$$

The choice of structure $\varphi(y)$ is given by the following modification of the theorem 3.7 [24].

Theorem 4. Let for the system (3) in the space $\mathscr{P}_{k_{s} e}=\left(k_{s}, e\right)$ :

i) the framework $S_{e k}$ and the secant described by the equation (14), are constructed;

ii) the order of the polynomial (14) satisfies the condition (15);

iii) the class $\mathcal{F}_{P_{f}}$ of elementary functions is set;

iv) the set of secants (16) for $S_{e k}$ is constructed on the basis of functions $P_{f_{n}} \in F_{P_{f}}, n=\overline{1, m_{T_{P_{f}}}}$ and the coefficient of determination $r_{e k, i, P_{f_{n}}}^{2}$ determined.

Then the structure of the function $\varphi(y)$ coincides with structure of the function $P_{f_{n}} \in F_{P_{f}}$, if

$$
\left|r_{e k, P_{f_{i}}}^{2}-\delta_{*}^{2}\right| \leq \Delta
$$

where $\Delta \geq 0$ is some magnitude, $\delta_{*}^{2}$ is determined from the condition (17).

Algorithm $A F_{o v}$ of a choice of the structure the func- tion $\varphi(y)$ on a class $F_{o v}$.

1. Construct the framework $S_{e y}$.

2. Perform the fragmentation $S_{e y}: S_{e y}=\bigcup_{i} F R_{\varphi}^{i}$.

3. Determine the structure $S_{e y}$ on the basis of the analysis of the fragments $F R_{\varphi}^{i} \subseteq S_{e y}$ and construct the sector $\operatorname{Sec}\left(F R_{\varphi}^{i}\right)$ for $F R_{\varphi}^{i} \subseteq S_{e y}$.

4. Estimate structural identifiability of a systemon $S_{e y}$.

5. Make the solution on the class $F_{o v}$. If $\varphi(y) \in F_{o v}$, go to the step 6 , otherwise, end the algorithm.

6. Go in structural space $\mathscr{P}_{k e}=\left(k_{s}, e\right)$ and will construct framework $S_{e k}$.

7. Select in $S_{e k}$ the fragments $F R_{\varphi, k}^{i}$ corresponding $F R_{\varphi}^{i} \subseteq S_{e y}$.

8. Consider the fragment $F R_{\varphi, k}^{i} \subset S_{e k}, i \geq 1$ on $S_{e k}$.

9. Determine the secant $\bar{\gamma}_{k, p}^{i}$ (14), and parameter $p_{i}$ select from the condition (15).

10. Set the class of the elementary single-valued functions $F_{P_{f}}$.

11. Construct the set of secants (16) on $F_{P_{f}}$ for $F R_{\varphi, k}^{i} \subset S_{e k}$ and compute the coefficients of determination $r_{e k, i, P_{f_{n}}}^{2}, n=\overline{1, m_{T_{P_{f}}}}$.

12. Apply the theorem 4 and obtain the structure of the function $\varphi(y)$.

Remark 5. The described approach to choose of secant in [18] is called the straightening method. The graphanalytic approach similar to this method was applied in [25].

Remark 6. We identified the form and parameters $\varphi(y)$ on the basis of the analysis of framework $S_{e k}$. Explain it to that the framework $S_{e k}$ unlike $S_{e y}$ gives real estimations of parameters the function $\varphi(y)$. The justification of application the framework $S_{e k}$ is given in [7, 19, 24].

Class $F_{m v}$

Consider a class of multiple-valued functions $F_{m v}$. Use frameworks $S_{e y}, S_{e k}$ for the problem solution.

Algorithm $A F_{m v}$ of the choice of the structure $\varphi(y)$ on a class $F_{m v}$.

1. Construct the framework $S_{e y}$.

2. Estimate on $S_{e y} h_{\delta_{h}}$-identifiability of the system(3). If the system (3) is structurally identified, go to the step 3, otherwise end the algorithm.

3. Part $S_{e y}$ into fragments $F R_{\varphi}^{i} \subseteq S_{e y}, i \geq 1$. 
4. Construct the sector $\operatorname{Sec}\left(F R_{\varphi}^{i}\right)$ for $F R_{\varphi}^{i}$. Make the solution on the class $\mathcal{F}_{m v}$. If $\varphi(y) \in F_{m v}$, go to the step 5, otherwise end of the algorithm.

5. Make the preliminary solution in the form of the function $\varphi(y)$ on the basis of the analysis $S_{e y}$.

6. Go in the structural space $\mathscr{P}_{k e}=\left(k_{s}, e\right)$ and will construct the framework $S_{e k}$.

7. Estimate properties and parameters of the function $\varphi(y)$ on the basis of the analysis frag ments $F R_{\varphi, k}^{i} \subset S_{e k}$, $i \geq 1$. Apply the secant method in space $\mathscr{P}_{k e}$.

8. Find for $F R_{\varphi, k}^{i}$ the secant $\bar{\gamma}_{i, k}$ and the coefficient of determination $r_{e, \bar{\gamma}_{i, k}}^{2}$ corresponding to it.

9. Make the solution on a significance of obtained parameters of function $\varphi(y)$ on $r_{e, \bar{\gamma}_{i, k}}^{2}$.

Remark 7. The proposed approach allows to obtain parametric estimations for $\bar{\gamma}_{i, k}$ in the form of some subset belonging to the informational set $\mathrm{I}_{o}$. The level of obtained values is one more measure of the adequacy of the proposed approach.

Remark 8. The algorith $m$ based on the analysis of frameworks $S_{e y}$ and $S_{e k}$ does not allow to identify any class of nonlinearities. The make the decision about the completeness of the algorithm $A F_{m v}$ or its modification follows only on the basis of the detailed analysis of the framework $S_{e y}$. Results of work [26] confirm this conclusion.

Remark 9. The system phase portrait $S$ can give the additional information on a system class. But this information is not such representative. It gives general concept of a system. The framework $S_{e y}$ is "cleared" and gives to the accented reflection of systemfeatures.

So, algorithms of the form identification the nonlinearity in the system (3) are proposed. Now perform the validation of obtained estimations. The first approach is based the parametric identification. It is very labourconsuming stage. It is not always simple realized in the conditions of a priori uncertainty. We propose the method of the structurally-frequency analysis (SFA). It is proposed in [16] for verification of results structural identification of a dynamic system with complicated piecewise constant nonlinearity. Further, we describe method SFA.

\section{G. SFA and Its Application}

Structurally-frequency analysis is based on the representation of image nonlinearity in the form of structurally-frequency chart (SFC). This diagram class is proposed in [16]. SFC is one of effective methods of decisionmaking under uncertainty. The frequency analysis is the development of the histogram method applied in statistics. We interpret the frequency of appearance the determined signal of the certain level as the indication of existing properties function. It is the fundamental diffe rence SFC from the accepted statistical interpretation of frequency.

SFC determines the relation between changes of argument $y$ and a response (function $\varphi(y))$ on this effect. The frequency of distribution $y$ can increase in some areas. It is the emersion indicator in a system of a nonstandard (nonlinear) regime. Distribution functions $y$ and $\varphi(y)$ have the equal definition domain. Therefore, the growth of frequency $n_{y}$ should confirm features of change $\varphi(y)$. These properties reflect SFC.

Remark 10. SFC can have a different form. Distribution laws $y, \varphi(y)$ depend on properties of a nonlinear system. As $y(t)$ is a continuous function almost for $\forall t \geq 0$ then $n_{e}$ (distribution $\left.\varphi(y)\right)$ has to a maximum in areas $J_{y}^{M} . J_{y, i}^{M}$ correspond to features of processes in a system. SFC can have several such areas.

Consider SFC as one more form of dynamic systems frameworks at decision-making level.

Name these distributions $S$-distributions to distinguish them from static distributions.

Example 3. Consider the system of the second order with parameters

$$
\begin{gathered}
A=\left[\begin{array}{cc}
0 & 1 \\
-3 & -4
\end{array}\right], y(0)=x(0)=2, \\
y^{\prime}(0)=x^{\prime}(0)=1, u(t)=3 \sin (0.1 \pi t), y=x, \\
\varphi(y)=\left\{\begin{array}{cc}
2, & \text { if } y>1, \\
\operatorname{sign}(y), & \text { if } y \leq 1, \\
-2, & \text { if } y<-1 .
\end{array}\right.
\end{gathered}
$$

where $\operatorname{sign}(y)$ is the $\operatorname{sign}$ function.

Show in Fig. 6 frameworks describing the steady state to this system. We see (Fig. 6) that the system is $h_{\delta_{h}}$ identified. The system has features in points $y=-1$, $y=0, y=1$. These points are points of the sign change the derivative (see $S_{e y}$-structure).

Identification of structural parameters of this system is considered in [16]. Therefore, go to the decision-making stage about nonlinearity of the system. Apply the method of the structurally-frequency analysis. Results show in Fig. 7. We see that SFC has two levels. The distribution function $f(y)$ of variable $y$ is presented at the top level. The distribution function $f(e)$ is shown at the lower level (the y-axis is had on the right). $f(e)$ has the complicated form and levels of its values are correlated with change $f(y)$. We represent at this level result decisionmaking on $\varphi(y)$. The restored configuration of $\varphi(y)$ in scale of the variable $e$ is presented as $\varphi_{e}(y)$. Show ar 
rows of points the switching of function $\varphi(y)$. We represent the fragment of the top piece of the framework $S_{e y}$ on the plane $(y, e)$.

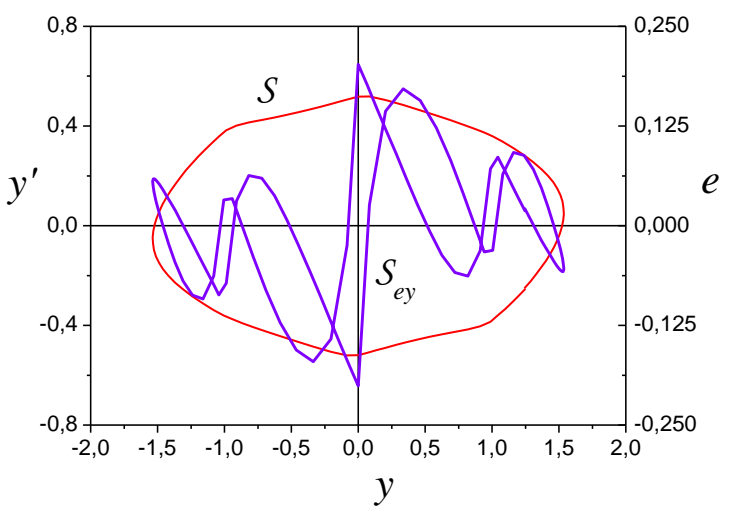

Fig.6. Frameworks of the second order system with the nonlinearity (18).

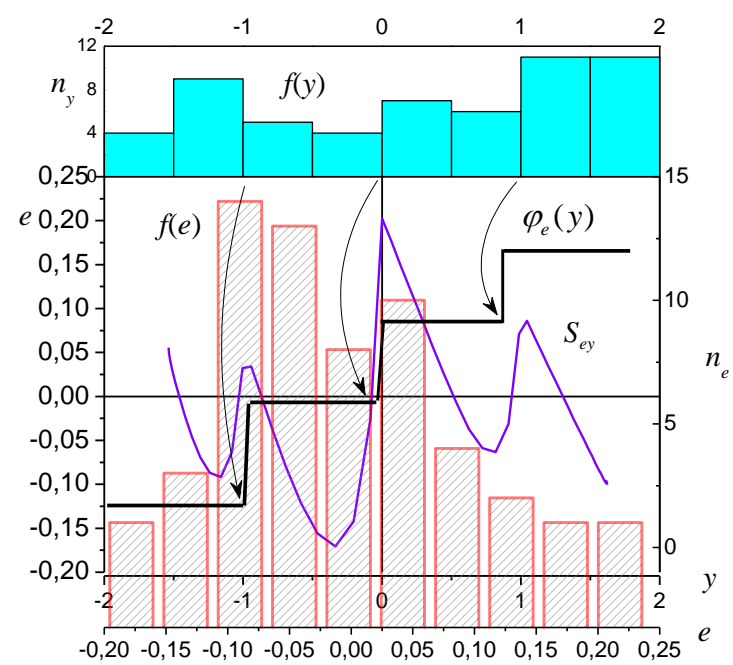

Fig.7. Results of application method SFA.

So, the method of SFA allows making the decision about the form $\varphi(y)$ on the basis of the analysis of functions $n_{e}(e), n_{e}(e)$ the framework $S_{e y}$. The given approach is demonstrative but demands good knowledge of object domain. Therefore, the combination of a priori knowledge and method SFA allow to obtaining the finished solution of the problem.

Remark 11. The structurally-frequency analysis is applied to the solution of various problems the analysis dynamic systems. The example 3 shows that the correct interpretation of results expands decision-making options in systems of structural identification. SFC is applicable also in identification system of eigenvalues dynamic system (see further).

Remark 12. Frameworks $S_{e y}$ also $S_{e k}$ solve special classes of problems. $S_{e y}$ is applied at decision making level about a form of nonlinearity and structural identifiability of a system. The framework $S_{e k}$ is a basis for an estimation of structural parameters nonlinearity.

The problem solution in the nonlinearity form gave to the application of frameworks $R \mathcal{K}_{v r}$ and $\mathcal{K}_{v \pm}$ [27]. These frameworks were proposed for estimation of hysteresis structure in static systems. Binary mapping $\mathscr{B}_{ \pm}$is proposed on the basis of the analysis of these frameworks. $\mathscr{B}_{ \pm}$describes the structure of hysteresis a logic function. Results of modeling show that $R \mathcal{K}_{v r}, \mathcal{K}_{v \pm}$ and the mapping $\mathscr{B}_{ \pm}$was applicable in systems of structural identification dynamic systems.

We noted that choice of form model (7) is the complicated problem. Show how to choose the model (7) for an obtaining of the framework $S_{e y}$ and to identify the form of nonlinearity.

Example 4. Let we had the information $\mathrm{I}_{o}$ on dynamic system. Show to the phase portrait $S$ of a system in the Fig. 8. We saw that processes in the system had complicated character.

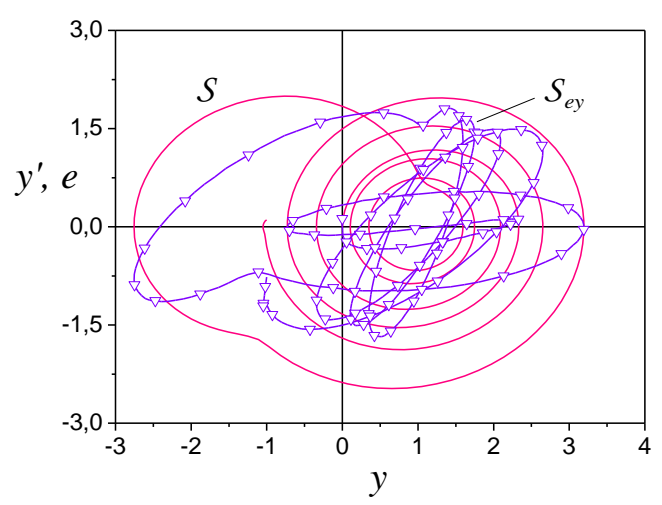

Fig.8. Frameworks $S, S_{e y}$.

Construct the model (7) and generate the variable $e(t)$ for $t \in[0 ; 7] \mathrm{s}$. Model (7) has the form

$$
\hat{x}_{1}^{l}(t)=[-0.12 ; 14.68 ; 0.1][1 u(t) y(t)]^{T}
$$

Coefficient of determination $r_{1}^{2}=0.97$. Construct the framework $S_{e y}$ and show to it in the Fig. 8. The framework $S_{e y}$ has the complicated form. We could not make the solution on the class of function $\varphi(y)$ by $S_{e y}$. Consider two hypotheses.

1. Model (7) is inadequate to the system.

2. Model (7) are not applicable for the specific class of systems.

We reject the hypothesis 1 , as the coefficient of determination is very high. Hence, the hypothesis 2 is true. The model had a more complex structure. Suppose that nonlinearity are described by a differential equation (compare $S_{e y}$ and $S$ ). Examine this hypothesis. 
Apply numerical differentiation and determine the second derivative $y(t)$. Designate the obtain variable as $x_{2}$. Consider the model

$$
\hat{x}_{2}^{l}(t)=H_{2}^{T}\left[\begin{array}{lll}
1 & u(t) & y(t)
\end{array}\right]^{T} .
$$

Let the variable $x_{2}$ is more informative, than $x_{1}$. Apply LSM and obtain

$$
\begin{gathered}
\hat{x}_{2}^{l}(t)=[0.961 ; 1.91 ;-0.982][1 u(t) y(t)]^{T}, \\
r_{2}^{2}=0.987 .
\end{gathered}
$$

The model (19) is determined for $t \in[0 ; 7.8] \mathrm{s}$. Introduce the variable $e_{2}(t)=\hat{x}_{2}^{l}(t)-x_{2}(t)$.

Show in Fig. 9 of frameworks reflecting the state of the identification system. Present in Fig. 9 the framework $S_{e_{2} y}$ described by mapping $\Gamma_{e_{2} y}:\{y\} \rightarrow\left\{e_{2}\right\}$.

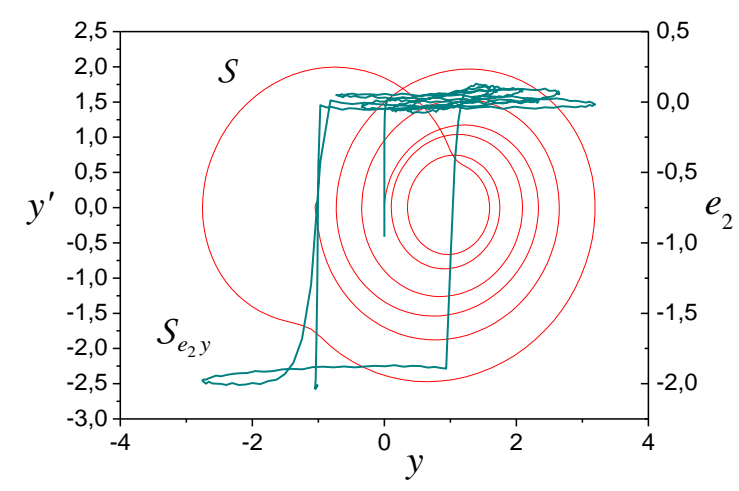

Fig.9. Frameworks $S, S_{e_{2} y}$.

Fig. 9 allows making the decision on the nonlinearity of the system. The solution gives to the analysis $S_{e_{2} y}$. $S_{e_{2} y}$ represents the modification of the ideal hysteresis. We see that the variable $e_{2}$ in the saturation range have small fluctuations. Analyze the nature of these fluctuations.

Assumption 1. Fluctuations are a property of a system or the impact of uncertainty which is the inadequacy of the model (19).

Apply model

$$
\hat{x}_{2(3)}^{l}(t)=H_{3}^{T}\left[\begin{array}{llll}
1 & u(t) & y(t) & x_{1}(t)
\end{array}\right]^{T}
$$

to verification of the as sumption 1 .

Obtain the estimation $H_{3}$ on the time gap $[0 ; 7.8] \mathrm{s}$. Vector $H_{3}=[0.99 ; 1,06 ;-1 ; 0,06]^{T}, r_{2}^{2}=0.996$. Let $e_{3}(t)=\hat{x}_{2(3)}^{l}(t)-x_{2}(t)$. Show in Fig. 10 to the framework $S_{e_{3} y}$ describing the change of the hysteresis $\varphi(y)$.

Compare $S_{e_{2} y}, S_{e_{3} y}$ and obtain what fluctuations of the framework $S_{e_{2} y}$ is the effect of the inadequacy of the model (19) in the saturation range. The model (20) eliminates these fluctuations. Show to change of in itial nonlinearity $\varphi(y)$ in Fig. 10. $S_{e_{3} y}$ and $\varphi(y)$ are congruent. The offset is the result of the transition in the structural space.

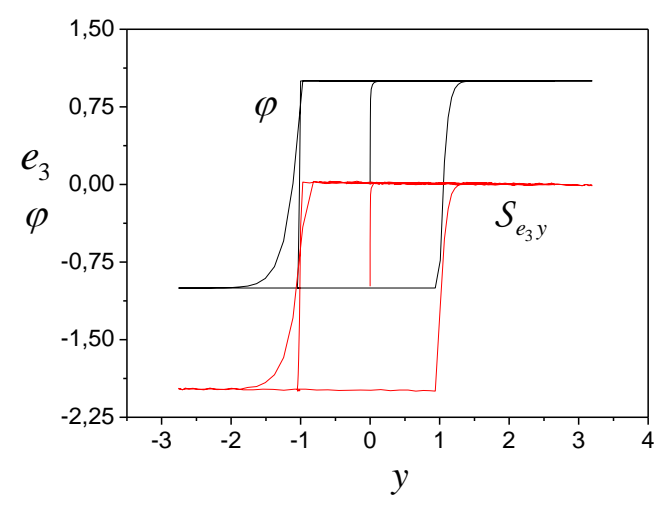

Fig.10. Hysteresis $\varphi(y)$ and its estimation $e_{3}(y)$.

We do not consider a problem of the time gap choice for identification of models (7), (19), (20). Choice of the interval depends on properties of a system and is defined at the modeling stage. Our goal is choice of frameworks allowing solving the problem of the nonlinearity class estimation. Further researches of this problem are given in [7]. They show that the examined nonlinearity is RCOTA the chaotic oscillator [28] described by system of equations

$$
\begin{aligned}
& \ddot{x}-0.1 \dot{x}+x=\varphi, \\
& \dot{\varphi}=10(-\varphi+\operatorname{sgn}(x+\operatorname{sgn}(\varphi))), \\
& y=x,
\end{aligned}
$$

Example 4 shows that the choice of the form equation (7) for obtaining of the framework $S_{e y}$ is the complex task. Properties of the nonlinear system define by the detail level of the model (7). If nonlinearity is described by a dynamic model, then the framework $S_{e y}$ obtained on the basis of the model (7), has the complex form. Its form complicates the decision-making on the structure of the nonlinearity. It is the indication on the search of new dependence for the identification $S_{e y}$ or its analogues. We understand under analogues virtual frameworks which determine relations between new variables. It is the main inference which gives to the example 4 .

\section{FRAMEWORKS IN IDENTIFICATION SYSTEMS OF LINEAR DYNAMIC SYSTEMS}

We will state to the approach to structural identification of linear dynamic systems based on application SA [29].

A. Problem Statement 
Consider linear dynamic system

$$
\begin{aligned}
& \dot{X}=A X+B U, \\
& Y=C X+D U,
\end{aligned}
$$

where $X \in R^{m}$ is a state vector, $U \in R^{k}, Y \in R^{n}$ are input and output, $A \in R^{m \times m}, B \in R^{m \times k}, C \in R^{n \times m}, D \in R^{n \times k}$.

The experimental information for (21) has the form

$$
\mathrm{I}_{o}=\left\{Y(t), U(t), t \in J=\left[t_{0}, t_{1}\right]\right\} .
$$

Write a solution of system(21) as

$$
X(t)=X\left(t_{0}, U, t\right)
$$

where $X$ is the operator uniquely defined by matrixes $A, B$.

Obtain the solution of the system (21) with $X_{0}=X\left(t_{0}\right)$

$$
X(t)=X_{g}(t)+X_{q}(t),
$$

where $X_{q}(t)$ is a particular solution (21) with $U \in \mathrm{I}_{o}$, $X_{g}(t)$ is the general solution (1) with $U(t)=0$ with an unknown vector $X_{0} \in \mathrm{I}_{o}$.

We designate the general solution (1) with $X_{0}=X_{0}\left(Y_{0}\right) \in \mathrm{I}_{o}$ as $X_{g}\left(X_{0}, t\right)$.

Problem: find the solution estimations $X_{g}(t)=X_{g}\left(X_{q}, X_{0}, t\right)$ on the set $\mathrm{I}_{o}$ and evaluate a spectrum of eigenvalues and the order of the system(21).

\section{B. Estimation $X_{g}(t)$}

Consider the special case of the system (21) with $Y=y, y \in R, U=u, u \in R$, and $m=2$. Apply approach, proposed in [18]. Generate the set $\left\{X_{g}(t)\right\}$, having applied operation $\{X(t)\} \backslash\left\{X_{q}(t)\right\}$. Present $\mathrm{I}_{o}$ as

$$
\mathrm{I}_{o}=\mathrm{I}_{o}^{q}\left(J_{q}\right) \cup \mathrm{I}_{o}^{g}\left(J_{g}\right),
$$

where $J_{q} \cup J_{g}=J \subseteq R ; \mathrm{I}_{o}^{q}, \mathrm{I}_{o}^{g}$ are sets which contain the information about $X_{q}$ and $X_{g}$.

Find the estimation of the particular solution the system (21) on the basis of the analysis $\mathrm{I}_{o}^{q}\left(J_{q}\right)$. As $x_{1}=y \in R$ apply operation of differentiation of the variable $y$ to obtaining the component $x_{2}=\dot{x}_{1}$ of the vector $X \in R^{2}$. Designate $x_{2}=\dot{y}$.

Statement 4 [18]. The model

$$
\hat{X}_{q}(t)=\hat{A}_{q} W(t) \quad \forall t \in J_{q},
$$

gives the estimate $X_{q}(t)$ on the set $\mathrm{I}_{o}^{q}$, where $\hat{A}_{q} \in R^{2 \times 2}$ is the matrix of parameters, $W=\left[\begin{array}{ll}u & u^{\prime}\end{array}\right]^{T}$.

Properties of the model (24) depend on the choice of an interval $J_{q} \subset J$. We determine by an estimation $\hat{X}_{q}(t)$ of a particular solution $X_{q}(t)$ of system (21) on the set $I_{o}^{g}$, having applied the model (24). Find the estimation of the general solution as

$$
\hat{X}_{g}(t)=X(t)-\hat{X}_{q}(t) \quad \forall t \in J_{g},
$$

where $\hat{X}_{g}(t)=\left[\hat{y}_{g}(t) \dot{\hat{y}}_{g}(t)\right]^{T}$.

The proposed approach can be generalized on a multidimensional case.

Further we consider the system (21) with one input $u$ and one output $y$.

The model (24) is applicable at the certain restrictions imposed on the input $u(t)$. The input should be constantly excited and limited, and the system (21) is $h$ identified.

Further, we use Lyapunov exponents and frameworks on their basis for structural identification of the system.

Remark 13. The considered problem has the specificity. Its solution demands introduction of the frameworks which are different from $S_{e y}$. These frameworks are based on Lyapunov exponents (LE) and a coefficient of structural properties.

\section{LE. Coefficient of Structural Properties}

Apply Lyapunov exponents [30] to the estimation of eigenvalues spectrum of the dynamic system(21). LE use as the system stability criterion. Determine LE for a realvalued function $h(t)$ as

$$
\chi[h]=\varlimsup_{t \rightarrow \infty} \frac{\ln |h(t)|}{t},
$$

where $\varlimsup_{t \rightarrow \infty}$ is a limit superior?

$\chi_{i} \quad(i=\overline{1, m})$ a non-zero solution of the system $(21)$ coincides with real parts of eigenvalues $\lambda_{i}$ of a matrix $A$.

Let the estimation of the general solution $X_{g}(t)$ $\forall t \in J_{g}$ of the system (21) is known and the system is stable. Apply (25) to $\hat{y}_{g}(t)$

$$
\chi\left[\hat{y}_{g}\right]=\varlimsup_{t \rightarrow \bar{t}} \frac{\ln \left|\hat{y}_{g}(t)\right|}{t},
$$

where $\bar{t} \in J_{g}$ is the maximum value $t$ on the interval $J_{g} \subset J$.

$\chi\left[\hat{y}_{g}\right]$ is the largest LE. If the limit (25) exists, 
$\chi\left[\hat{y}_{g}\right]$ is the estimation of the largest eigenvalue of the matrix $A$. So, $\chi\left[\hat{y}_{g}\right]$ is an index of degree stability the system(21). If $m=2$, for $\dot{\hat{y}}_{g}$ obtaining

$$
\chi\left[\dot{\hat{y}}_{g}\right]=\varlimsup_{t \rightarrow \bar{t}} \frac{\ln \left|\dot{\hat{y}}_{g}\right|}{t} .
$$

Consider the index

$$
\eta[h]=\varliminf_{t \rightarrow \infty} \frac{\ln |h(t)|}{t},
$$

where $\lim _{t \rightarrow \infty}$ is a bottom limit. It is the Perron bottom index [25]. Further, we will describe the estimation method $\eta[h]$.

The idea of application LE in problems of structural identification is proposed in $[18,32]$. The approach is based on the analysis of the coefficient of structural properties (CSP).

Introduce the index

$$
\rho\left(\hat{y}_{g}\right)=\rho_{g}(t)=\ln \left|\hat{y}_{g}(t)\right| \quad \forall t \in \bar{J}_{g} \subset J_{g} .
$$

$\bar{J}_{g}=\left[t_{0}, \bar{t}\right]$ determine on the basis of (26).

Consider the system with the input $t$ and the output $\rho\left(\hat{y}_{g}\right)$. Define CSP as

$$
k_{s}(t, \rho)=\frac{\rho\left(\hat{y}_{g}\right)}{t} .
$$

$k_{s}(t, \rho)$ is a basis for computing of the index $\chi\left[\hat{y}_{g}\right]$ on the interval $\bar{J}_{g}$.

We consider $k_{s}(t, \rho)$ as a transmission coefficient of a system with an input $t$ and the output $\rho\left(\hat{y}_{g}\right)$. Further, we describe the application $k_{s}(t, \rho)$ for the estimation LE.

So, the relationship between LE $\chi\left[\hat{y}_{g}\right]$ and $k_{s}(t, \rho)$ is shown. These indexes are defined on the informational set $\mathrm{I}_{\rho}=\left\{\rho\left(\hat{y}_{g}(t)\right), t \in \bar{J}_{g}\right\}$.

Consider the set

$$
\mathrm{I}_{g}\left(\hat{y}_{g}, t\right)=\left\{\hat{y}_{g}(t), t \in J_{g}\right\}=\mathrm{I}_{\hat{x}_{g}} \backslash\left\{\dot{\hat{y}}_{g}(t) t \in J_{g}\right\},
$$

It contains the data about the change variable of $\hat{y}_{g}$ on the interval $J_{g}$.

Assumption 2. The system (21) is stable, that is $\operatorname{Re}\left(\lambda_{i}\right) \leq 0, i=\overline{1, m}$, where $\lambda_{i} \in \sigma(A)$ is $i$-th eigenvalue of matrix $A$.
Problem: estimate the spectrum $\sigma(A)$ of eigenvalues of the matrix $A$ and an order of the system (21) on the basis of the analysis set $I_{g}$.

\section{Frameworks for Estimation LE}

Consider sets

$$
\mathrm{I}_{k_{s}}=\left\{k_{s}\left(t, \rho\left(\hat{y}_{g}(t)\right)\right), t \in \bar{J}_{g}\right\},
$$

$$
\mathrm{I}_{k_{s}^{\prime}}=\left\{k_{s}\left(t, \rho\left(\dot{\hat{y}}_{g}(t)\right)\right), t \in \bar{J}_{g}\right\} .
$$

Define on $\mathrm{I}_{k_{s}}, \mathrm{I}_{k_{s}^{\prime}}$ mapping $S_{k_{s, p}} \rightarrow \mathrm{I}_{k_{s}} \times \mathrm{I}_{k_{s}^{\prime}}$. The frame work $S_{k_{s, \rho}}$ reflects the dynamics of change parameters depending from $\mathrm{LE}$. Consider on set $\mathrm{I}_{k_{s}^{\prime}}$ function

$$
\Delta k_{s}^{\prime}(t)=k_{s}\left(t, \rho\left(\dot{\hat{y}}_{g}(t+\tau)\right)\right)-k_{s}\left(t, \rho\left(\dot{\hat{y}}_{g}(t)\right)\right)
$$

where $\tau>0$.

Generate the set $\mathrm{I}_{\Delta k_{s}^{\prime}}=\left\{\Delta k_{s}\left(t, \rho\left(\dot{\hat{y}}_{g}(t)\right)\right), t \in \bar{J}_{g}\right\}$ and introduce the framework $S \mathcal{K}_{\Delta k_{s, \rho}^{\prime}} \rightarrow \mathrm{I}_{k_{s, \rho}} \times \mathrm{I}_{\Delta k_{s, \rho}^{\prime}}$. Consider corresponding $S K_{\Delta k_{s, \rho}^{\prime}}$ the mapping

$$
\mathcal{L} S \mathcal{K}_{\Delta k_{s, \rho}^{\prime}} \rightarrow \mathrm{I}_{k_{s, \rho}} \times B\left(\mathrm{I}_{\Delta k_{s, \rho}^{\prime}}\right)
$$

where $B\left(\mathrm{I}_{\Delta k_{s, \rho}^{\prime}}\right) \subset\{-1 ; 1\}$. Define elements of the binary set $B\left(\mathrm{I}_{\Delta k_{s, \rho}^{\prime}}\right)$ as

$$
b(t)=\left\{\begin{array}{r}
1, \text { if } \Delta k_{s}^{\prime}(t) \geq 0, \\
-1, \text { if } \Delta k_{s}^{\prime}(t)<0,
\end{array} \quad t \in \bar{J}_{g} .\right.
$$

Remark 14. We can choose for some class of systems of boundary the limit superior in (26) on the basis of the change analysis $S_{k_{s, \rho}}$.

Remark 15. We have defined a choice of the range of values the function $b(t)$ by convenience of its graphical analysis. $b(t)$ is possible to specify on the binary set $\{0$; $1\}$.

\section{E. Estimation of System Order}

Consider criterion for the estimation of the system order (21) on the basis of the properties analysis of the framework $\mathcal{L} S \mathcal{K}_{\Delta k_{s, \rho}^{\prime}}[29]$.

Theorem 5. If the function $b(t)$ changes the sign $m-1$ of times on the interval $\left[t_{0}, t^{*}\right] \subset \bar{J}_{g}\left(t^{*} \leq \bar{t}\right)$, then the system(21) has an order $m$.

The theorem 5 is fair for multiple roots and simpler e i 
genvalues of the matrix $A$ system(21).

So, application of structural methods allows obtaining the estimation for the order systems (21). Theorem 5 has sufficient character and is applicable not to all types of roots. If conditions of the theorem 5 not are fulfilled, then use the algorithm of decision making proposed in [18].

Remark 16. The algorith $m$ for the estimation the order of a system under indeterminacy is proposed in [18]. It based on the analysis CSP the system (21). The approach described here is simple to application.

\section{F. Decision-making about Type of Roots}

The criterion for decision-making about a type of roots is proposed in [18]. It is based on the analysis of the variation the function $d(t)=\rho$ (疼) $/ \rho\left(\dot{y}_{g}\right)$. If the system has imaginary or complex eigenvalues, then apply other approaches and additional criteria. We propose the approach based on the analysis of special frameworks.

As shown in [29], system eigenvalues influence on form of the frame work $S$. Therefore, we introduce a special framework for decision-making about a spectrum $\sigma(A)$ of the system. The framework analysis gives to estimations of numbers $\lambda_{i}$.

Consider frameworks $S K_{k_{s, \rho}^{i}} \rightarrow \mathrm{I}_{k_{s}} \times \mathrm{I}_{k_{s}^{i}}$ and $\mathcal{L} S K_{\Delta k_{s, \rho}^{\prime}}$, where $i$ designate $i$-th derivative $\hat{y}_{g}(t)$,

$$
\mathrm{I}_{k_{s}^{i}}=\left\{k_{s}\left(t, \rho\left(\hat{y}_{g}^{(i)}(t)\right)\right), t \in \bar{J}_{g}\right\} .
$$

The largest Lyapunov exponent $\chi_{l}\left[\hat{y}_{g}\right]$ is located in the left part of the framework $S K_{k_{s, \rho}^{i}}$. All other indexes $\chi_{i}\left[\hat{y}_{g}\right]$ coincide with Perron bottom indices $\eta_{i}\left[\hat{y}_{g}\right]$ and are located to the right from $\chi_{l}\left[\hat{y}_{g}\right] \cdot \chi_{i}\left[\hat{y}_{g}\right]$ correspond to local minima on $S K_{k_{s, \rho}^{i}}$. The disposition of minima on $S K_{k_{s, \rho}^{i}}$ coincides with the change of sign $\mathcal{L} S K_{\Delta k_{s, \rho}^{\prime}}$. We obtain the set $\mathcal{M}_{L E}=\left\{\hat{\lambda}_{1}, \hat{\lambda}_{2}, \ldots, \hat{\lambda}_{m}\right\}$, where $\hat{\lambda}_{i}$ is the estimation $i$-th eigenvalue $\lambda_{i}$ of the matrix $A$ system (21).

Theorem 6 [29]. If the system (21) is stable and has simpler eigenvalues, then frameworks $S K_{k_{s, \rho}^{i}}, i=\overline{1, m}$ contain the information on Lyapunov exponents.

We consider stable system (21), therefore, we make the solution only for negative values $\mathrm{I}_{k_{s}}$.

Apply the method of the histogram to confirmation of the obtained solution. It is special case SFA, described in section 3.G. Construct for each informational set $\mathrm{I}_{k_{s}}, \mathrm{I}_{k_{s}^{i}}$ histograms $\mathcal{H}_{k_{s}}, \mathcal{H}_{k_{s}^{i}}$ showing distribution of obtained indexes $\chi_{i}\left[\hat{y}_{g}\right]$. The maximum value of the function
$\mathcal{H}_{k_{s}^{i}}$ is the index $\chi_{l}\left[\hat{y}_{g}\right]$, and the minimum values are $\chi_{i}\left[\hat{y}_{g}\right]$. These results are one more confirmation of Lyapunov exponents classification for a dynamic system.

Remark 17. Use properties of a framework $\mathcal{L} S K_{\Delta k_{s, \rho}^{\prime}}$ for an elimination "false" LE in $\mathcal{H}_{k_{s}}, \mathcal{H}_{k_{s}^{i}}$.

\section{G. Procedure of Definition LE}

The proposed methods and algorithms is an approach basis to structural identification of linear dynamic systems. Generalizing the obtained results, we propose procedure of structural identification. It is based on the co mputing LE. The proposed approach gives estimations of type LE and of the linear system dimension.

\section{Procedure CLE.}

1. Generate sets for computing LE. Use the results obtained in section 4.B. Apply statement 4 and obtain the variable $X_{g}(t)$.

2. Construct the phase portrait of the system (21) and check the $h$-identifiability.

3. Determine system coefficient of structural properties on the set $\mathrm{I}_{g}\left(\hat{y}_{g}, t\right)$.

4. Construct frameworks $\mathcal{L} S K_{\Delta k_{s, \rho}^{\prime}}, S K_{\Delta k_{s, \rho}^{\prime}}$.

5. Estimate the system order. Analyze $\mathcal{L} S K_{\Delta k_{s, \rho}^{\prime}}$ and apply the theorem 5 .

6. Analyze the function $d(t)$ and make the solution on roots type of the system (21). Perform the analysis of frameworks $S K_{k_{s, \rho}^{i}}$.

7. Fulfil check of the correctness of obtained estimations on the basis of the construction a special class of frequency distributions.

Example 5. Consider the system(21) with parameters

$$
\begin{gathered}
A=\left[\begin{array}{ccc}
0 & 1 & 0 \\
0 & 0 & 1 \\
-6 & -11 & -6
\end{array}\right], \sigma(A)=(-1 ;-2 ;-3), \\
y(0)=y^{\prime \prime}(0)=1, y^{\prime}(0)=2 \\
u(t)=3+2 \sin (0.1 \pi t), y=x_{1} .
\end{gathered}
$$

Apply the approach proposed in section 4.B, and obtain the set $\left\{\hat{y}_{g}(t), 0 \leq t \leq 16 \mathrm{~s}\right\}$. Generate sets $\mathrm{I}_{\Delta k_{s}^{\prime}}, \mathrm{I}_{k_{s}}$, $\mathrm{I}_{k_{s}^{i}}, i=1,2$ on the basis of processing $\hat{y}_{g}(t)$. Apply the theorem 5 and present results of the estimation of the system order and eigenvalues of the matrix $A$ in Fig. 11. 


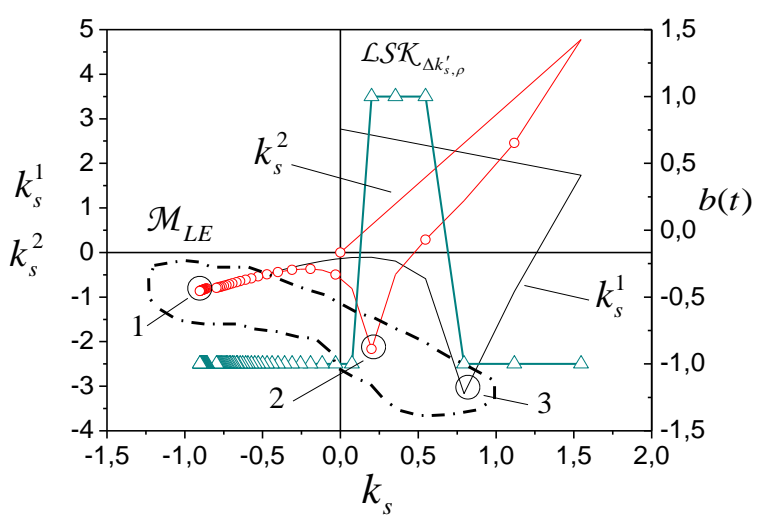

Fig.11. Estimation of the order and eigenvalues of the system.

We allocated set $\mathcal{M}_{L E}$ in Fig. 11 and designated estimations $\lambda_{i}, i=1,2,3$ as $1,2,3$. We see that the framework $\mathcal{L} S K_{\Delta k_{s, \rho}^{\prime}}$ changes a sign twice. Consequently, the system has the third order. Apply following designations in Fig. 11

$$
k_{s}=k_{s}(t, \rho), k_{s}^{1}=k_{s}\left(t, \rho\left(\hat{\dot{y}}_{g}(t)\right)\right), k_{s}^{2}=k_{s}\left(t, \rho\left(\ddot{\hat{y}}_{g}(t)\right)\right) .
$$

Construct bar graphs of distributions of numbers $\hat{\lambda}_{i}$ (Fig. 12) for check of the correctness of the obtained solutions. Show to frequencies $f_{i}$ of values hit $k_{s}, k_{s}^{1}, k_{s}^{2}$ at the specified intervals (pockets) and the set $\mathcal{M}_{L E}$ in Fig. 12. As the system is stable, we will consider frequencies $f_{i}$ only for negative values $k_{s}^{i}$. Obtain following set $\mathcal{M}_{L E}=\{-0.9 ;-2.16 ;-3.16\}$ of eigenvalues. Except value -4 on the basis of the remark 17 .

Example 6. Consider the system (21) the second order with $\sigma(A)=(-2 i ; 2 i)$. Input $u(t)=3+2 \sin (0.1 \pi t)$, $y(0)=2, y^{\prime}(0)=1$. The condition $h$-identifiability is fulfilled. We do not represent the form of the system matrix $A$. It has the form of the Frobenius matrix.

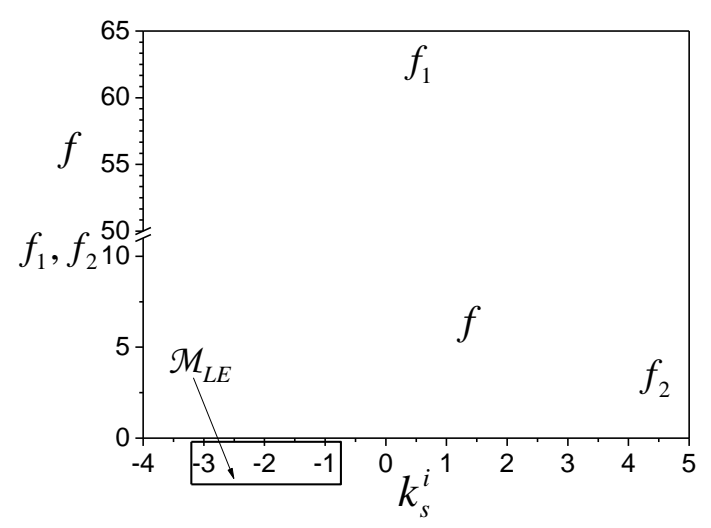

Fig. 12. Distribution of simple roots of the third order system.

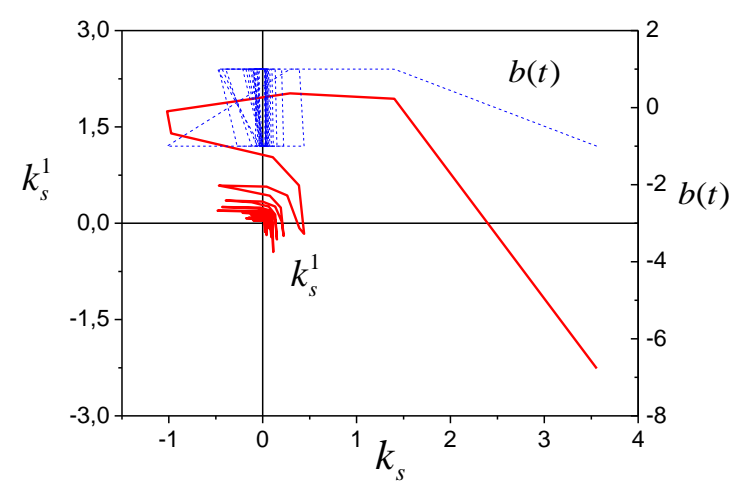

Fig.13. Estimation of imaginary roots for system of the second order.

Show to results of the application of frameworks and procedure CLE in Fig. 13. We see that the system does not have real roots. The system order is equal to two. The framework $S K_{k_{s, \rho}}$ has oscillation about coordinate orig in with descending $k_{s}$. It is an indication of presence of imaginary roots. The largest LE on the bar graph (Fig. 14) is equal to zero. It coincides with the initial assumption. The method of the estimation of the imaginary part of roots by means of secants is described in [19].

Example 7. Consider the system (21) of second order with

$$
\begin{gathered}
A=\left[\begin{array}{cc}
0 & 1 \\
-2 & -2
\end{array}\right], \sigma(A)=(-1+i,-1-i), \\
u(t)=5+2 \sin (0.1 \pi t), y(0)=2, y^{\prime}(0)=1 .
\end{gathered}
$$

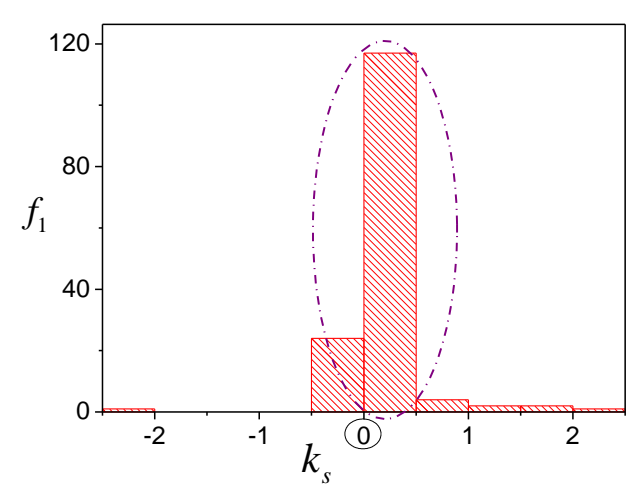

Fig.14. Distribution of imaginary roots of the second order system.

Results of identification are presented in Fig. 15, 16. 


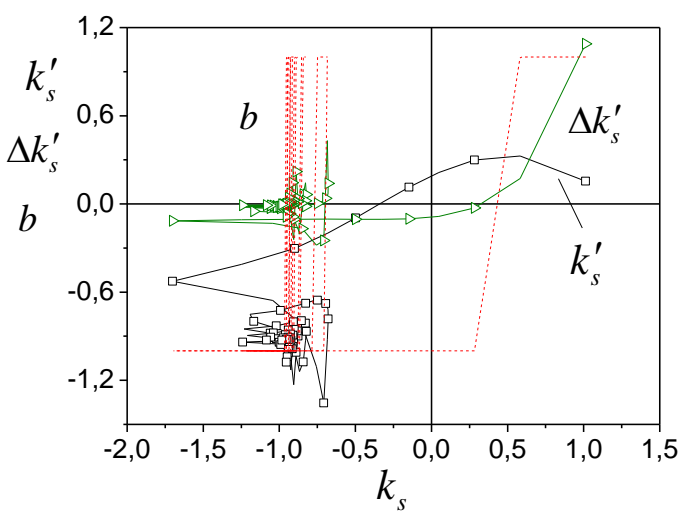

Fig.15. Frameworks for the estimation of the system order with complex $\operatorname{roots}(m=2)$

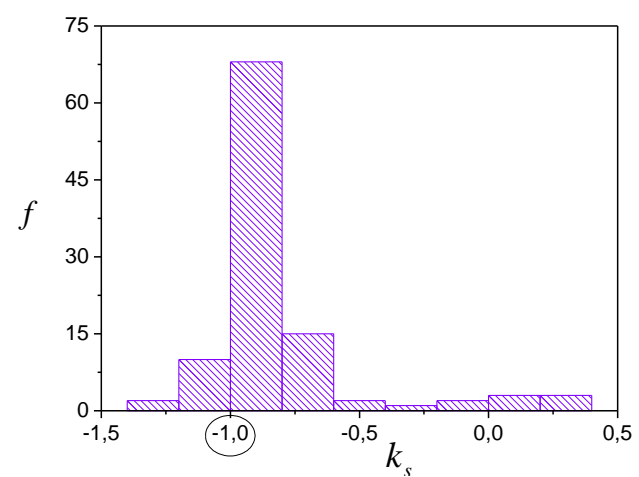

Fig.16. Distribution of imaginary roots of the second order system.

The analysis of the variation $b(t)$ (Figure 15) gives the estimation of the system order: $m=2$. We observe oscillations with decreasing amplitude around value $k_{s}(t, \rho)=-1 . k_{s}(t, \rho)=-1$ is the value of the real part of a root. So, the framework $\mathcal{L} S \mathcal{K}_{\Delta k_{s, \rho}^{\prime}}$ confirms presence of complex roots.

The function $f$ of distribution values $k_{s}$ is shown in Fig. 16. It confirms the presence of the complex root and shows to oscillations around value -1 .

So, results of modeling confirm workability and efficiency of proposed structural methods and procedures of linear dynamic systemidentification.

\section{Struct URAL Methods in Nonlinear St atiC SYSTEM IDENTIFICATION}

Ideas and the methods stated in sections 1-3 have been verified for the first time on static systems (SS) [19, 22]. Static systems have the specificity. They do not have an equilibrium state. Therefore, the framework $S_{e y}$ in these conditions is ineffective. Often inputs SS are irregular (random). Therefore, application of frameworks $S_{e y}$, in this case, is not effectively. Development was obtained by the methods of structural identification based on the analysis $S_{e k}$-structures. Procedure of data ordering in SS on the coefficient of structural properties is applied to an elimination of effect of an irregularity and not measured noises. We considered above application $S_{e k}$ for decision-making on the structure of a system. $S_{e k}$ gives to the solution of the estimation problem of nonlinearity structural parameters $[22,26,33]$ in $\mathrm{SS}$. The secant method is the basis of analysis algorithms $S_{e k}$. The set of secants specifies the field of frameworks SS.

Remark 18. The secant method is used as an analysis method $S_{e y}$-structures of dynamic systems.

The main advantage the secant method is decisionmaking on a class of linear functions (secants). The secant method gives to the problem identification solution of parametric restrictions SS under uncertainty [34]. The special class of frameworks specified in space of system outputs and model outputs, is applied to the estimation of parametric restrictions. The secant method is applicable for estimation of the linearity SS [24].

\section{CONCLUSION AND DISCUSSION}

The analysis of approaches to construction and application of frameworks in systems of nonlinear dynamics and control is performed. Application of frame works gives to the problem solution of an estimation qualitative behavior nonlinear systems. Many researchers will understand frameworks as mathematical objects or the mappings describing features of processes in a system. The bas is of the structural approach in control systems is mappings having geometrical representation. These representations give to processes in the systemto a visual interpretation.

We interpret the structural approach as construction and the analysis of geometrical frame works which reflect features of the system. The structural approach in such interpretation in identification problems was not applied. Review of works shows that at identification problems solving the concept "the structural approach" is widely applied. This concept of identification systems does not coincide with interpretation in nonlinear dynamics. It reflects the content of a research area. Such interpretation of the structural approach reduces the solution of the structural identification problem to the application of parametric methods. It eliminates essential properties of a dynamic system. Hence, we make the conclusion about the necessity of application untraditional identification procedures.

We give to generalization and development the $S I$ approach on structural identification problems of nonlinear dynamic systems. The basis $S I$-approach is virtual frameworks $S_{e y}$, reflecting properties of the nonlinear part of the system. Methods of formation of an auxiliary informational set are considered. This set is the bas is for construction $S_{e y}$. Models for the formation of the virtual framework $S_{e y}$ are proposed. The construction problem such models is actual. The model choice is defined by properties and structure of the nonlinear dynamic system. We consider examples of the model structure choice, making the analys is of system properties. As there are 
many nonlinear dynamic systems, general approach to design of such models does not exist.

Introduction VF demands design of estimation methods of the structural identifiability nonlinear dynamic systems. Structural identifiability defines a possibility of the framework estimation of a nonlinearity. Criteria of the structural identifiability estimation are proposed. These criteria determine de mands to the form $S_{e y}$. Conditions of emergence "insignificant" frameworks are considered. These frameworks are not are structurally identified. The concept $D_{h}$-dimensions of nonlinear dynamic system is introduced. We will show if the system is structurally identifiable, then it $D_{h}$-dimension it is close to the identity.

We propose criteria of decision-making about the class of functions describing nonlinearity in the dynamic system. Clas ses of single-valued and multiple-valued nonlinearities are considered. Algorithms and methods of the structure estimation of single-valued and multiple-valued nonlinearities are proposed. The verification of the obtained structural solutions is fulfilled by means of the method of the structurally -frequency analys is proposed in work. The method is applicable under uncertainty. We apply also frameworks $S_{e k}$. $S_{e k}$ give to the solution of such problems as the choice of the nonlinearity form and its structural parameters.

We show that virtual frameworks are applicable in the structure estimation problems of linear dynamic systems. Specificity VF for this class of systems is shown. VF allows identify ing the order and the eigenvalues spectrum of the system. Virtual frameworks describe a change LE. Frequency charts are used for the check of the obtained estimations of the system eigenvalues spectrum. Frequency charts are the special case of structurally-frequency charts.

We generalize $S I$-approach on static nonlinear systems. Features VF for the given class of systems are noted. We show that the main role play $S_{e k}$-frameworks. They give to the solution of structural identification problem in a special space.

So, the method of virtual frameworks allows to solving various problems of structural identification. Approaches to construction VF are defined by the available a priori and experimental information. Construction $\mathrm{VF}$ under uncertainty is a complex problem. Identification of a structural feature of the system defines the form VF. The correctness of the obtained virtual framework is verified on the basis of the analysis of its geometrical image.

\section{REFERENCES}

[1] S.N. Rasband, Chaotic Dynamics of Nonlinear Systems. Mineola, New York: Dover Publications, INC, 2015.

[2] Advances and Applications in Chaotic Systems. Volume 6. Ed. S. Vaidy anathan, Ch. Volos. Springer, 2016.

[3] N.N. Bautin, E.A. Leontovich, Methods and techniques of qualitative research of dynamic systems on a plane. Moscow: Nauka, 1990.

[4] V.N. Shuman, Nonlinear dynamics of geomedium: transitional processes and critical phenomena, Geophysical journal, 2014, vol. 36, no. 6, pp. 129-141.

[5] D.V. Anosov, "Roughness of geodesic flows on compact Riemannian manifolds of negative curvature," Dokl. Akad. Nauk SSSR, 1962, vol. 145, pp. 707-709.

[6] V.V. Gorbatsevich, "On Algebraic Anosov Diffeomorphisms on Nilmanifolds," Siberian Mathematical Journal, 2004, vol. 45 , is. 5, pp. 821-839.

[7] N. Karabutov, "Structural Identification of Dynamic Sy stems with Hysteresis," International journal of intelligent systems and applications, 2016, vol. 8, no. 7, pp. 1-13.

[8] K.F. Alvin, A.N. Robertson, G.W. Reich, K.C. Park, "Structural system identification: from reality to models," Computers and Structures, 2003, vol. 81, pp. 1149-1176.

[9] R. Pasquier, I. F. C. Smith, "Iterative structural identification framework for evaluation of existing structures," Engineering Structures, 2016, vol. 106, pp. 179-194.

[10] A. Hyvärinen, K. Zhang, S. Shimizu, P.O. Hoyer, "Estimation of a Structural Vector Autoregression Model Using Non-Gaussianity," Journal of Machine Learning Research, 2010, vol. 11, pp. 1709-1731.

[11] G.F. Sirca Jr., H. Adeli, "System identification in structural en gineering," Scientia Iranica A, 2012, vol. 19, no. 6, pp. 1355-1364.

[12] S. Yamada, A. Nishitani, "Structural sy stem identification based on subsystem analyses," In 12WCEE 2000: 12th World Conference on Earthquake Engineering, Auckland, New Zealand, Sunday 30 January - Friday 4 February 2000, 2000, pp. 1-8.

[13] V. Namdeo, C.S. Manohar, "Nonlinear structural dynamical system identification using adaptive particle filters," Journal of Sound and Vibration, 2007, vol. 306, pp. 524563.

[14] G. Kerschen, K. Worden, A.F. Volakis, J.C. Galingale, "Nonlinear Sy stem Identification in Structural Dy namics: Current Status and Future Directions," https://www.google.ru/url?sa=t\&rct=j\&q=\&esrc=s\&sourc $\mathrm{e}=$ web\&cd=48\&ved $=0$ ahUKEwiHmaCg4prM AhURKy w KHfhkCuU4KBAWCEcwBw\&url=http s\%3A\%2F\%2For bi.ulg.ac.be $\% 2$ Fbitstream $\% 2 \mathrm{~F} 2268 \% 2 \mathrm{~F} 22625 \% 2 \mathrm{~F} 1 \% 2 \mathrm{FC}$ 28_2007_Orlando_IMAC_CurrentStatus.pdf\&usg=AFQj CNHm9X4eHh3YEc70RnkRZVFZmzGcpQ\&sig2=GOB BSFeI-

we3oUksAbALt1g\&bvm=bv.119745492,d.bGg\&cad=rjt, 2007.

[15] N.N. Karabutov, "Structural identification of nonlinear static system on basis of analysis sector sets," International journal intelligent systems and applications, 2013, vol. 6, no. 1, pp. 1-10.

[16] N. Karabutov, "Structural Identification of Nonlinear Dynamic Systems," International journal intelligent systems and applications, 2015, vol, 7, no.9, pp. 1-11.

[17] N.N. Karabutov, Adaptive identification of systems: Informational synthesis. Moscow: URSS, 2016.

[18] N.N. Karabutov, Structural Identification of Systems. The Analysis of Information Structures. URSS/ Librokom, Moscow, 2016.

[19] N.N. Karabutov. Structural identification of static objects: Fields, structures, methods. Moscow: Librokom, 2016.

[20] Y.E., Kazakov B.G. Dostupov, Statistical dy namics of nonlinear automatic systems. Moscow: Fizmatgis, 1962.

[21] V.D. Furasov, Stability of motion, estimation and stabilization. Moscow: Nauka, 1977.

[22] N. Karabutov, "Structures, Fields and Methods of Identification of Nonlinear Static Systems in the Conditions of Uncertainty," Intelligent Control and Automation, 2010, vol.1 no.2, pp. 59-67.

[23] G. Choquet, L'enseignement de la geometrie. Hermann, 
Paris, 1964.

[24] N. Karabutov, Methods of structural identification of nonlinear objects. Saarbrucken: Palmarium Academic Publishing, 2014.

[25] F. Mosteller, J. Tukey, Data analysis and regression: A second course in statistics. Addison-Wesley Publishing Company. 1977.

[26] N. Karabutov, "Structural identification of static processes with hysteresis nonlinearities in civil engineering," Journal of Civil Engineering and Science, 2012, vol. 1, no. 4, pp. 22-29.

[27] N. Karabutov, "About structures of state systems identification of static object with hysteresis," International journal of sensing, computing \& control, 2012, vol. 2, no. 2, pp. 59-69.

[28] L. Acho, "Hysteresis modeling and synchronization of a class of RC-OTA hysteretic-jounce-chaotic oscillators," Universal Journal of Applied Mathematics, 2013, vol. 1, n0. 2, pp. 82-85.

[29] N. Karabutov, "Structural Methods of Estimation Lyapunov Exponents Linear Dy namic System," International journal intelligent systems and applications, 2015, vol, 7, no.10, pp. 1-11.

[30] B.F. Bylov, R.E. Vinograd, D.M. Grobman, and V.V. Nemytsky, Theory of Lyapunov indexes and its application to stability problems. Nauka: Moscow, 1966.

[31] N.A. Izobov, Introduction in theory of Lyapunov indexes. Minsk: BGU, 2006.

[32] N.N. Karabutov, "Deriving a dynamic system eigenvalue spectrum under conditions of uncertainty by processing measurements," Measurement Techniques, 2009, vol. 52, no. 6, pp. 571-579.

[33] N. Karabutov, "Decision-making on structure of univ alent nonlinearities in system of structural identification of static systems," International journal of sensing, computing \& control, 2011, vol. 1, no. 2, pp. 103-110.

[34] N. Karabutov, "Identification of parametrical restrictions in static systems in conditions of uncertainty," International journal of intelligent systems and applications, 2013, vol. 5, no. 4, pp. 43-54.

\section{Authors' Profiles}

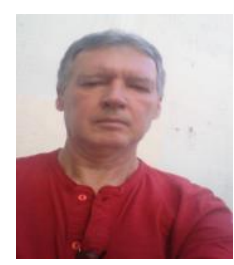

Nikolay Karabutov is the professor of department Problem Control of Moscow technological University (Moscow state engineering university of information technology, radio engineering, electronics), Moscow, Russia. Doctor of technical sciences, professor.

The research areas are the automatic control theory, identification, adaptive control and decision-making.

How to cite this paper: Nikolay Karabutov,"Frameworks in Problems of Structural Identification Systems", International Journal of Intelligent Systems and Applications(IJISA), Vol.9, No.1, pp.1-19, 2017. DOI: 10.5815/ijisa.2017.01.01 